data_CRYSTALS_cif of 3a

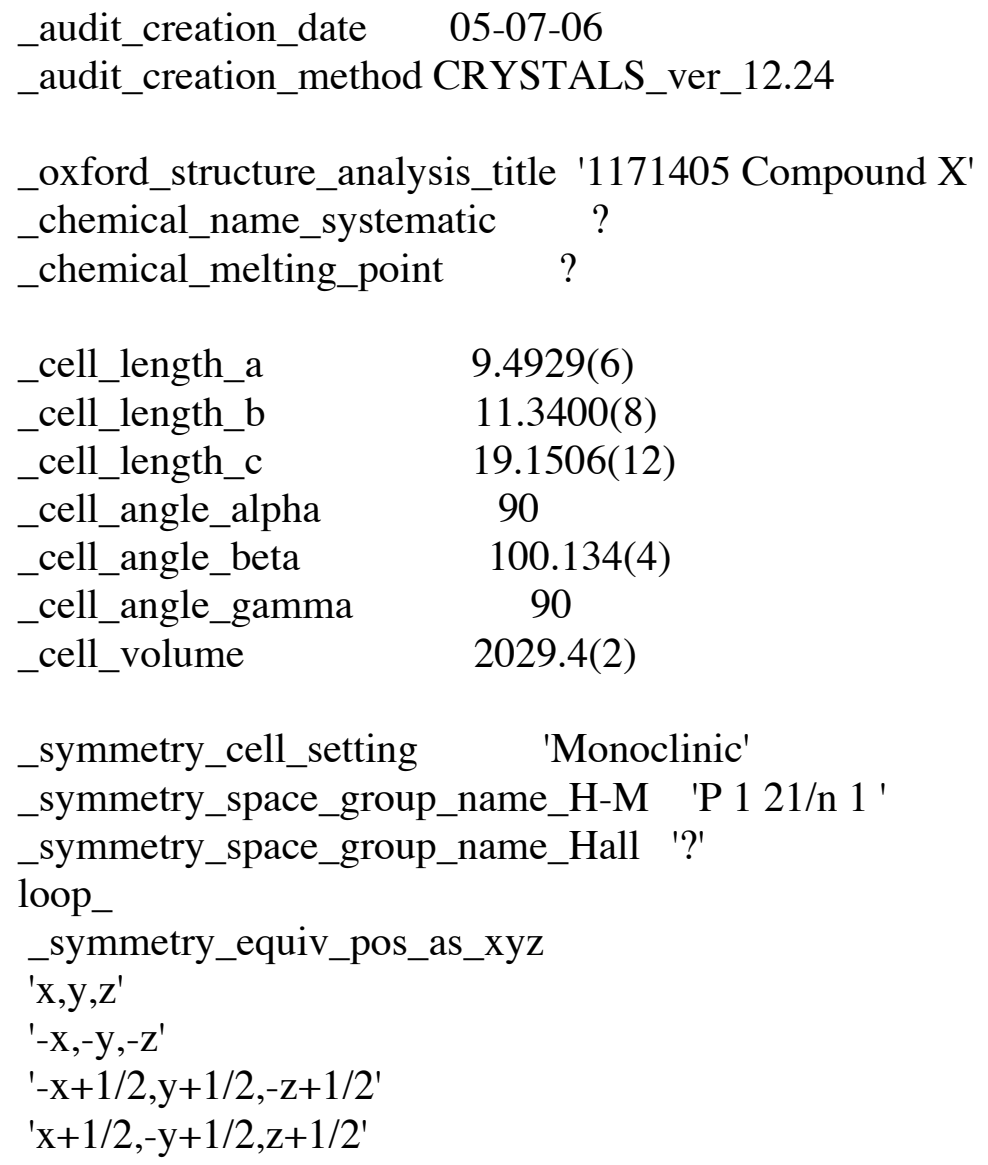

loop_

_atom_type_symbol

_atom_type_scat_dispersion_real

_atom_type_scat_dispersion_imag

_atom_type_scat_Cromer_Mann_a1

_atom_type_scat_Cromer_Mann_b1

_atom_type_scat_Cromer_Mann_a2

_atom_type_scat_Cromer_Mann_b2

_atom_type_scat_Cromer_Mann_a3

_atom_type_scat_Cromer_Mann_b3

_atom_type_scat_Cromer_Mann_a4

_atom_type_scat_Cromer_Mann_b4

_atom_type_scat_Cromer_Mann_c

_atom_type_scat_source

C $\quad \begin{array}{lllllllll}0.0033 & 0.0016 & 2.3100 & 20.8439 & 1.0200 & 10.2075 & 1.5886 & 0.5687\end{array}$

0.865051 .65120 .2156 'International Tables Vol C 4.2.6.8 and 6.1.1.4'

$\begin{array}{lllllllll}\mathrm{H} & 0.0000 & 0.0000 & 0.4930 & 10.5109 & 0.3229 & 26.1257 & 0.1402 & 3.1424\end{array}$

0.0408 57.7998 0.0030 'International Tables Vol C 4.2.6.8 and 6.1.1.4'

$\begin{array}{lllllllll}\mathrm{O} & 0.0106 & 0.0060 & 3.0485 & 13.2771 & 2.2868 & 5.7011 & 1.5463 & 0.3239\end{array}$

$0.867032 .9089 \quad 0.2508$ 'International Tables Vol C 4.2.6.8 and 6.1.1.4'

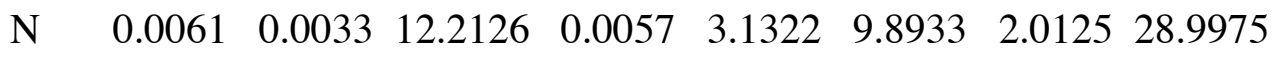

1.16630 .5826 -11.5290 'International Tables Vol C 4.2.6.8 and 6.1.1.4'

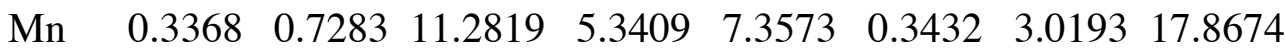

2.244183 .75431 .0896 'International Tables Vol C 4.2.6.8 and 6.1.1.4' 
_cell_formula_units_Z

4

_chemical_formula_sum

'C21 H15 Mn1 O5 S1'

_chemical_formula_moiety

'C21 H15 Mn1 O5 S1'

_chemical_compound_source

$?$

_chemical_formula_weight

434.34

_cell_measurement_reflns_used 80

_cell_measurement_theta_min 4

ccell_measurement_theta_max 21

_cell_measurement_temperature 295

_exptl_crystal_description_ 'parallelepiped'

_exptl_crystal_colour_yellow'

_exptl_crystal_size_min $\quad 0.08$

_exptl_crystal_size_mid $\quad 0.10$

_exptl_crystal_size_max $\quad 0.11$

_exptl_crystal_density_diffrn 1.422

_exptl_crystal_density_meas ?

\# Non-dispersive $\mathrm{F}(000)$ :

_exptl_crystal_F_000_ 888

_exptl_absorpt_coefficient_mu 0.782

_exptl_absorpt_correction_type multi-scan

_exptl_absorpt_process_details 'SADABS (Siemens, 1996)'

_exptl_absorpt_correction_T_min 0.92

_exptl_absorpt_correction_T_max 0.92

\# Sheldrick geometric definitions 0.920 .92

_diffrn_measurement_device_type 'Nonius Kappa CCD'

_diffrn_radiation_monochromator 'graphite'

_diffrn_radiation_type_ 'Mo Kla'

_diffrn_radiation_wavelength $\quad 0.71073$

_diffrn_measurement_method Iw

_computing_data_collection 'COLLECT (Nonius BV, 1997)'

_computing_cell_refinement 'Dirax/lsq (Duisenberg \& Schreurs, 1989-2000)'

_computing_data_reduction 'EvalCCD (Duisenberg \& Schreurs 1990-2000)'

_computing_structure_solution 'SHELXS 86 (Sheldrick, 1986)'

_computing_structure_refinement 'CRYSTALS (Betteridge et al 2003)'

_computing_publication_material 'CRYSTALS (Betteridge et al 2003)'

_computing_molecular_graphics 'CAMERON (Watkin et al 1996)'

_diffrn_standards_interval_time?

_diffrn_standards_interval_count? 
_diffrn_standards_number
_diffrn_standards_decay_\%

_diffrn_reflns_theta_min 2.256

_diffrn_reflns_theta_max 27.503

_diffrn_measured_fraction_theta_max 0.995

_diffrn_reflns_theta_full 26.953

_diffrn_measured_fraction_theta_full 0.996

\begin{tabular}{|c|c|}
\hline & -1 \\
\hline _diffrn_reflns_limit_h_max & 12 \\
\hline _diffrn_reflns_limit_k_min & \\
\hline _diffrn_reflns_limit_k_max & \\
\hline ddiffrn_reflns_limit_1_min & \\
\hline _diffrn_reflns_limit_1_max & \\
\hline _reflns_limit_h_min & -12 \\
\hline _reflns_limit_h_max & 12 \\
\hline _reflns_limit_k_min & 0 \\
\hline _reflns_limit_k_max & 14 \\
\hline _reflns_limit_1_min & 0 \\
\hline reflns_limit_1_max & 24 \\
\hline
\end{tabular}

_atom_sites_solution_primary direct \#heavy,direct,difmap,geom

\#_atom_sites_solution_secondary difmap

_atom_sites_solution_hydrogens geom

_refine_diff_density_min $\quad-0.34$

_refine_diff_density_max $\quad 0.38$

_refine_ls_number_reflns 2124

_refine_ls_number_restraints 1

_refine_ls_number_parameters 254

\#_refine_1s_R_factor_ref 0.0392

_refine_ls_wR_factor_ref 0.0433

_refine_ls_goodness_of_fit_ref 1.1362

\#_reflns_number_all 4628 
_refine_ls_R_factor_all 0.1058

_refine_ls_wR_factor_all 0.0451

\# The $\mathrm{I} / \mathrm{u}(\mathrm{I})$ cutoff below was used for refinement as

\# well as the _gt R-factors:

_reflns_threshold_expression I $>3.00 \mathrm{u}(\mathrm{I})$

_reflns_number_gt 2124

_refine_ls_R_factor_gt 0.0392

_refine_ls_wR_factor_gt 0.0433

_refine_1s_shift/su_max $\quad 0.001007$

\# choose from: rm (reference molecule of known chirality),

\# ad (anomolous dispersion - Flack), rmad (rm and ad),

\# syn (from synthesis), unk (unknown) or . (not applicable).

_chemical_absolute_configuration '.'

_refine_ls_structure_factor_coef $\mathrm{F}$

_refine_ls_matrix_type full

_refine_ls_hydrogen_treatment refU \# none, undef, noref, refall, \# refxyz, refU, constr or mixed

_refine_ls_weighting_scheme calc

_refine_ls_weighting_details

;

Method, part 1, Chebychev polynomial, (Watkin, 1994, Prince, 1982)

[weight $\left.]=1.0 /[\mathrm{A} \sim 0 \sim * \mathrm{~T} \sim 0 \sim(\mathrm{x})+\mathrm{A} \sim 1 \sim * \mathrm{~T} \sim 1 \sim(\mathrm{x}) . . .+\mathrm{A} \sim \mathrm{n}-1 \sim]^{* \mathrm{~T}} \sim \mathrm{n}-1 \sim(\mathrm{x})\right]$

where $\mathrm{A} \sim \mathrm{i} \sim$ are the Chebychev coefficients listed below and $\mathrm{x}=$ Fcalc/Fmax

Method $=$ Robust Weighting (Prince, 1982)

$\mathrm{W}=[$ weight $] *\left[1-\left(\text { deltaF } / 6^{*} \operatorname{sigmaF}\right)^{\wedge} 2^{\wedge}\right]^{\wedge} 2^{\wedge}$

A $\sim \mathrm{i} \sim$ are:

$2.61-2.382 .55-0.8340 .468$

;

\# Check this file using the IUCr facility at:

\# http://journals.iucr.org/services/cif/checking/checkfull.html

\# The content below is held in the file 'script/refcif.dat'. This is a text

\# file which you may edit to reflect local conditions.

\# Items which need looking at are represented by a '?'.

\# Items for which there are choices are prefixed with 'choose from'.

_publ_contact_letter

; Please consider this CIF submission for publication as a Short Format Paper in Acta

Crystallographica $\mathrm{C}$. The figures have been sent by mail. 
_publ_contact_author_name 'David Watkin'

_publ_contact_author_address

; Chemical Crystallography Laboratory,

Department of Chemistry,

University of Oxford,

Chemistry Research Laboratory,

Mansfield Road,

Oxford, OX1 3TA, UK.

;

_publ_contact_author_phone '+44 1865 285024'

_publ_contact_author_fax '+44 1865285018 '

_publ_contact_author_email_david.watkin@chem.ox.ac.uk'

_publ_requested_journal_Organometallics'

_publ_requested_category CO \# choose from: FI FM FO CI CM CO AD

_publ_requested_coeditor_name 'Prof William Clegg'

_publ_section_title \# Title of paper

; ?

;

\# The loop structure below should contain the names and addresses of all \# authors, in the required order of publication. Repeat as necessary.

loop_

_publ_author_name

_publ_author_address

_publ_author_footnote

'Other, Anthony N.' \# Author 1

; ? \# Address for author 1

;

; ? \# Footnote for author 1

;

'Else, S. O.' \# Author 2

; ? \# Address 2

;

; ? \# Footnote 2

;

_publ_section_abstract \# Text of the abstract

; ?

;

_publ_section_comment \# Text of the paper

; ?

_publ_section_acknowledgements \# Acknowledgments

; ?

;

_publ_section_figure_captions \# Captions to figures

; ?

; 
_publ_section_exptl_refinement \# see also_refine_ls_hydrogen

; $\mathrm{H}$ atoms placed geometrically after each cycle

_publ_section_exptl_prep

; ?

;

\# PROCESSING SUMMARY (IUCr Office Use Only):

_journal_date_recd_electronic ?

_journal_date_to_coeditor ?

_journal_date_from_coeditor ?

_journal_date_accepted?

_journal_date_printers_first ?

journal_date_printers_final ?

journal_date_proofs_out?

_journal_date_proofs_in?

_journal_coeditor_name?

_journal_coeditor_code?

_journal_coeditor_notes ?

_journal_techeditor_code?

_journal_techeditor_notes?

_journal_coden_ASTM?

journal_name_full ?

journal_year ?

_journal_volume ?

_journal_issue ?

_journal_page_first ?

_journal_page_last ?

_journal_suppl_publ_number ?

_journal_suppl_publ_pages ?

\# End of 'script/refcif.dat'

\# Insert your own references if required - in alphabetical order

_publ_section_references

;

Betteridge, P.W., Carruthers, J.R., Cooper, R.I.,

Prout, K., Watkin, D.J. (2003). J. Appl. Cryst. 36, 1487.

Nonius BV, COLLECT Software, (1997-2001)

Otwinowski, Z. \& Minor, W. (1996), Processing of X-ray

Diffraction Data Collected in Oscillation Mode. Methods Enzymol. 276, 1997, 307-326. Ed Carter, C.W. \& Sweet, R.M., Academic Press.

Prince, E. 
Mathematical Techniques in Crystallography

and Materials Science

Springer-Verlag, New York, 1982.

Siemens Industrial Automation, Inc (1996).

SADABS: Area-Detector Absorption Correction;: Madison, WI.

Sheldrick, G.M. (1986). SHELXS86. Program for the solution of crystal structures. Univ. of Gottingen, Federal Republic of Germany.

Watkin D.J. (1994),

Acta Cryst, A50, 411-437

Watkin, D.J., Prout, C.K. \& Pearce, L.J. (1996) CAMERON, Chemical Crystallography Laboratory, OXFORD, UK.

;

\# Uequiv $=$ arithmetic mean of Ui i.e. Ueqiv $=(\mathrm{U} 1+\mathrm{U} 2+\mathrm{U} 3) / 3$

\# Replace last . with number of unfound hydrogen atoms attached to an atom.

\# ..._refinement_flags_...

\# . no refinement constraints $\quad \mathrm{S}$ special position constraint on site

\# $\mathrm{G}$ rigid group refinement of site $\mathrm{R}$ riding atom

\# D distance or angle restraint on site T thermal displacement constraints

\# U Uiso or Uij restraint (rigid bond) P partial occupancy constraint

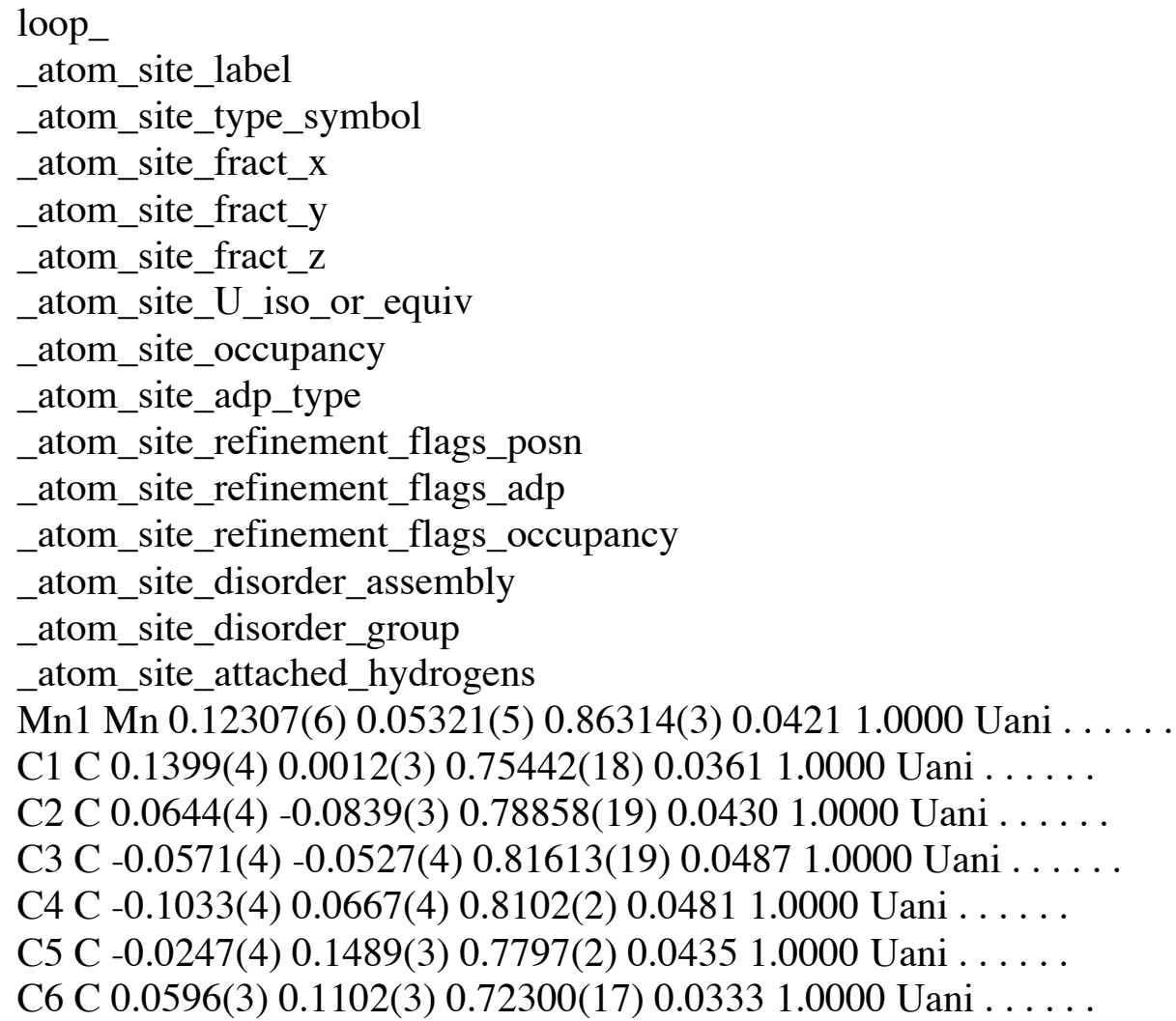


C7 C 0.2827(4) -0.0349(3) 0.74212(18) 0.04041 .0000 Uani . . . . . . C8 C 0.3592(4) 0.0312(3) 0.6934(2) 0.04111 .0000 Uani . . . . . . C9 C 0.3177(4) 0.1106(4) 0.6393(2) 0.04571 .0000 Uani . . . . . . C10 C 0.4311(5) 0.1455(4) 0.6053(3) 0.06411 .0000 Uani ... . . . C11 C 0.5549(6) 0.0938(5) 0.6334(4) 0.08701 .0000 Uani ....... C12 C -0.0313(3) 0.0978(3) 0.64900(18) 0.03651 .0000 Uani . . . . . C13 C -0.0692(4) -0.0097(4) 0.6173(2) 0.05071 .0000 Uani . . . . . C14 C -0.1486(5) -0.0141(4) 0.5487(2) 0.06181 .0000 Uani ...... C15 C -0.1894(5) 0.0876(5) 0.5122(2) 0.06711 .0000 Uani . . . . . . C16 C -0.1513(5) 0.1940(5) 0.5432(3) 0.06711 .0000 Uani . . . . . . C17 C -0.0727(4) 0.2005(4) 0.6116(2) 0.05441 .0000 Uani ....... C18 C -0.2603(7) 0.2077(5) 0.8468(4) 0.10511 .0000 Uani D . . . . C19 C 0.0664(5) 0.1055(4) 0.9428(2) 0.05961 .0000 Uani . . . . . . C20 C 0.2460(5) -0.0465(4) 0.9169(2) 0.06171 .0000 Uani ...... C21 C 0.2571(5) 0.1655(4) 0.8619(2) 0.04961 .0000 Uani . . . . . . O1 O -0.2122(3) 0.0915(3) 0.84541(18) 0.07061 .0000 Uani D . . . . O2 O 0.3442(3) $-0.1202(3) 0.77379(15) 0.05901 .0000$ Uani . . . . . . O19 O 0.0310(4) 0.1408(4) 0.99291(18) 0.09211 .0000 Uani . . . . . O20 O 0.3221(5) -0.1084(4) 0.9523(2) 0.10371 .0000 Uani . . . . . . O21 O 0.3436(4) 0.2348(3) 0.85905(17) 0.07701 .0000 Uani . . . . . . S1 S 0.53844(13) -0.00049(14) 0.70044(10) 0.09061 .0000 Uani ...... H21 H $0.1008-0.16790 .79340 .082(4) 1.0000$ Uiso . . . . . H31 H $-0.1116-0.11310 .83980 .082(4) 1.0000$ Uiso . . . . . . H51 H $-0.02460 .23410 .79500 .082(4) 1.0000$ Uiso . . . . . . H61 H $0.13350 .17330 .71980 .082(4) 1.0000$ Uiso . . . . . . H91 H $0.21590 .14030 .62540 .082(4) 1.0000$ Uiso . . . . . . H101 H $0.42010 .20420 .56430 .082(4) 1.0000$ Uiso . . . . . . H111 H $0.64850 .10860 .61630 .082(4) 1.0000$ Uiso . . . . . . H131 H -0.0391 -0.0856 $0.64420 .082(4) 1.0000$ Uiso . . . . . . H141 H $-0.1770-0.09320 .52540 .082(4) 1.0000$ Uiso . . . . . . H151 H $-0.24810 .08390 .46260 .082(4) 1.0000$ Uiso . . . . . . H161 H $-0.18070 .26920 .51530 .082(4) 1.0000$ Uiso . . . . . . H171 H $-0.04580 .28040 .63460 .082(4) 1.0000$ Uiso . . . . . . H181 H $-0.34180 .21500 .87370 .082(4) 1.0000$ Uiso . . . . . H182 H $-0.18190 .26300 .86820 .082(4) 1.0000$ Uiso . . . . . . H183 H -0.2975 0.2371 0.7961 0.082(4) 1.0000 Uiso . . . . . loop_

_atom_site_aniso_label _atom_site_aniso_U_11 _atom_site_aniso_U_22 _atom_site_aniso_U_33 _atom_site_aniso_U_23 _atom_site_aniso_U_13 _atom_site_aniso_U_12 Mn1 0.0504(3) 0.0398(3) 0.0364(3) $-0.0020(3) 0.0086(2)-0.0058(3)$ C1 0.0416(19) 0.0301(17) 0.0362(17) -0.0036(15) 0.0053(15) 0.0002(16) C2 0.054(2) 0.033(2) 0.0403(19) -0.0032(15) 0.0031(17) -0.0047(17) C3 0.053(2) 0.046(2) 0.048(2) -0.002(2) 0.0127(18) -0.015(2) C4 0.041(2) 0.055(3) 0.050(2) -0.009 (2) 0.0122(17) -0.0023(19) C5 0.045(2) 0.038(2) 0.047(2) -0.0045(17) 0.0090(17) 0.0000(17) 
C6 0.0320(17) 0.0288(18) 0.0387(17) 0.0011(15) 0.0052(14) 0.0016(14) C7 0.0418(19) 0.035(2) 0.0419(18) -0.0099(16) -0.0007(16) 0.0000(16)

C8 0.0322(17) 0.035(2) 0.056(2) -0.0125(16) 0.0065(16) -0.0011(15)

C9 0.041(2) 0.052(2) 0.047(2) -0.0002(19) 0.0143(17) -0.0029(18)

C10 0.064(3) 0.062(3) 0.073(3) -0.009(2) 0.033(2) -0.007(2)

C11 0.062(3) 0.077(4) 0.136(5) $-0.005(3) 0.055(3)-0.014(3)$

C12 0.0285(17) 0.0385(19) 0.0429(19) 0.0057(16) 0.0071(15) 0.0031(15)

C13 0.046(2) 0.049(2) 0.053(2) -0.0047(19) -0.0023(19) 0.0038(19)

C14 0.057(3) 0.072(3) 0.052(2) $-0.015(2)-0.003(2)-0.002(2)$

C15 0.059(3) 0.092(4) 0.044(2) 0.005(2) $-0.008(2)-0.004(3)$

C16 0.058(3) 0.076(3) 0.060(3) 0.023(3) -0.009 (2) 0.000(3)

C17 0.048(2) 0.051(3) 0.060(2) 0.012(2) $-0.0034(19)-0.004(2)$

C18 0.088(4) 0.097(5) 0.143(6) 0.001(4) 0.057(4) 0.021(4)

C19 0.070(3) 0.059(3) 0.052(2) $-0.007(2) 0.016(2)-0.015(2)$

C20 0.076(3) 0.057(3) 0.048(2) -0.001(2) 0.001(2) 0.005(3)

C21 0.053(2) 0.054(3) 0.040(2) -0.0056(18) 0.0050(18) -0.007(2)

O1 0.0571(18) 0.079(2) 0.084(2) -0.0112(18) 0.0358(17) 0.0007(16)

O2 0.0622(18) 0.0447(17) 0.0674(18) 0.0087(14) 0.0042(15) 0.0191(14)

O19 0.123(3) 0.097(3) 0.068(2) -0.028(2) 0.050(2) -0.021(2)

$\mathrm{O} 200.120(3) 0.105(3) 0.074(2) 0.022(2)-0.014(2) 0.032(3)$

$\mathrm{O} 210.078(2) 0.079(2) 0.070(2)-0.0079(18) 0.0036(17)-0.038(2)$

S1 0.0408(6) 0.0877(10) 0.1452(14) 0.0205(10) 0.0214(7) 0.0134(6)

_refine_ls_extinction_method

'None'

loop

_geom_bond_atom_site_label_1

_geom_bond_site_symmetry_1

_geom_bond_atom_site_label_2

_geom_bond_site_symmetry_2

_geom_bond_distance

_geom_bond_publ_flag

Mn1 . C1 . 2.197(3) yes

Mn1.C2.2.119(3) yes

Mn1.C3 . 2.153(4) yes

Mn1.C4.2.214(4) yes

Mn1.C5. 2.215(4) yes

Mn1 . C19. 1.805(4) yes

Mn1 . C20 . 1.810(5) yes

Mn1 . C21 . 1.804(4) yes

C1.C2 . 1.428(5) yes

C1.C6.1.519(5) yes

C1.C7.1.474(5) yes

C2.C3.1.396(5) yes

$\mathrm{C} 2$. H21. 1.011 no

C3. C4 . 1.422(6) yes

C3 . H31 . 1.012 no

C4.C5.1.385(5) yes

$\mathrm{C} 4 . \mathrm{O} 1.1 .360(5)$ yes

C5 . C6. 1.522(5) yes 
C5 . H51. 1.009 no

C6. C12 . 1.531(5) yes

C6. H61 . 1.012 no

C7 . C8 . 1.482(5) yes

C7. O2. 1.233(4) yes

C8.C9 . 1.377(5) yes

C8.S1 . 1.721(4) yes

C9. C10.1.409(6) yes

C9. H91 . 1.013 no

C10.C11.1.339(7) yes

C10.H101.1.022 no

C11.S1 . 1.699(6) yes

C11.H111. 1.013 no

C12.C13 . 1.381(5) yes

C12.C17.1.387(5) yes

C13.C14. 1.396(6) yes

C13 . H131 . 1.017 no

C14.C15. 1.368(7) yes

C14. H141 . 1.017 no

C15.C16. 1.365(7) yes

C15 . H151 . 1.014 no

C16.C17. 1.391(6) yes

C16. H161 . 1.017 no

C17. H171 . 1.020 no

C18.O1.1.396(6) yes

C18. H181 . 1.005 no

C18. H182 . 1.005 no

C18. H183 . 1.028 no

C19. O19.1.143(5) yes

$\mathrm{C} 20 . \mathrm{O} 20.1 .141(5)$ yes

C21. O21.1.144(5) yes

loop_

_geom_angle_atom_site_label_1

_geom_angle_site_symmetry_1

_geom_angle_atom_site_label_2

_geom_angle_site_symmetry_2

_geom_angle_atom_site_label_3

_geom_angle_site_symmetry_3

_geom_angle

_geom_angle_publ_flag

C1 . Mn1 . C2 . 38.59(14) yes

C1 . Mn1 . C3 . 68.83(13) yes

C2 . Mn1 . C3 . 38.15(15) yes

C1 . Mn1 . C4 . 79.11(13) yes

C2 . Mn1 . C4 .67.87(15) yes

C3 . Mn1 . C4 . 37.96(15) yes

C1 . Mn1 . C5 .65.81(13) yes

C2 . Mn1 . C5 . 79.36(14) yes

C3 . Mn1 . C5 . 67.42(15) yes

C4 . Mn1 . C5 . 36.45(14) yes 
C1 . Mn1 . C19 . 166.33(18) yes

C2 . Mn1 . C19.136.79(18) yes

C3 . Mn1 . C19.101.75(17) yes

C4 . Mn1 . C19.87.42(18) yes

C5 . Mn1 . C19. 101.77(18) yes

C1 . Mn1 . C20 . 102.95(16) yes

C2 . Mn1 . C20 . 89.51(17) yes

C3 . Mn1 . C20 . 105.95(19) yes

C4 . Mn1 . C20 . 141.30(19) yes

C5 . Mn1.C20. 168.20(16) yes

C1 . Mn1. C21 . 90.45(15) yes

C2 . Mn1 . C21 . 127.72(16) yes

C3 . Mn1 . C21 . 153.98(16) yes

C4 . Mn1.C21 . 125.10(17) yes

C5 . Mn1 . C21 .90.18(16) yes

C19. Mn1 . C20 . 89.0(2) yes

C19. Mn1.C21.95.46(19) yes

C20.Mn1.C21.93.6(2) yes

Mn1.C1.C2 .67.74(19) yes

Mn1.C1.C6. 92.4(2) yes

C2 . C1 . C6 . 118.3(3) yes

Mn1.C1.C7 . 116.6(2) yes

C2.C1.C7 . 115.8(3) yes

C6.C1.C7 . 125.0(3) yes

Mn1.C2.C1.73.7(2) yes

Mn1.C2.C3 .72.3(2) yes

C1.C2.C3 . 121.1(3) yes

Mn1.C2 . H21. 125.778 no

C1.C2 . H21 . 119.158 no

C3 . C2 . H21 . 119.769 no

Mn1.C3 . C2 . 69.6(2) yes

Mn1.C3 .C4 .73.3(2) yes

C2.C3 .C4 . 118.4(3) yes

Mn1.C3.H31. 128.382 no

C2 . C3 . H31 . 121.244 no

C4.C3 . H31. 120.383 no

Mn1.C4 .C3 .68.7(2) yes

Mn1.C4.C5 .71.8(2) yes

C3 .C4.C5 . 119.5(3) yes

Mn1.C4.O1.123.3(3) yes

C3.C4.O1 . 114.1(4) yes

C5.C4.O1.125.7(4) yes

Mn1.C5.C4.71.7(2) yes

Mn1.C5.C6.91.6(2) yes

C4 . C5 . C6 . 119.8(3) yes

Mn1.C5 . H51. 106.976 no

C4 . C5 . H51 . 119.627 no

C6. C5 . H51 . 120.616 no

C5 . C6 . C1 . 104.0(3) yes

C5 . C6.C12.113.9(3) yes 
C1.C6.C12.117.0(3) yes

C5 . C6 . H61 . 107.239 no

C1. C6 . H61 . 107.140 no

C12.C6 . H61 . 107.002 no

C1 .C7 . C8 . 121.8(3) yes

C1.C7.O2 . 120.6(3) yes

C8 . C7.O2 . 117.6(3) yes

C7.C8.C9 . 134.2(3) yes

C7.C8.S1.115.6(3) yes

C9.C8.S1.110.1(3) yes

C8 . C9 . C10 . 112.9(4) yes

C8.C9.H91. 123.289 no

C10 . C9 . H91 . 123.766 no

C9 . C10 . C11 . 112.3(4) yes

C9 . C10 . H101 . 123.711 no

C11.C10.H101 . 123.952 no

C10.C11.S1 . 112.8(4) yes

C10 . C11 . H111 . 123.771 no

S1 . C11. H111. 123.399 no

C6 . C12 . C13 . 123.3(3) yes

C6.C12.C17. 117.6(3) yes

C13 . C12 . C17 . 119.1(3) yes

C12 . C13 . C14 . 120.0(4) yes

C12 . C13 . H131 . 119.723 no

C14.C13.H131. 120.241 no

C13 . C14 . C15 . 120.5(4) yes

C13 . C14 . H141 . 120.064 no

C15 . C14 . H141 . 119.411 no

C14 . C15 . C16 . 119.6(4) yes

C14 . C15 . H151 . 120.159 no

C16 . C15 . H151 . 120.221 no

C15 . C16.C17 . 120.8(4) yes

C15 . C16 . H161 . 119.030 no

C17.C16. H161. 120.138 no

C16.C17.C12 . 119.9(4) yes

C16 . C17 . H171 . 120.342 no

C12.C17.H171. 119.752 no

O1.C18 . H181 . 111.772 no

O1 . C18 . H182 . 112.050 no

H181 . C18 . H182 . 108.676 no

O1.C18. H183 . 110.319 no

H181 . C18 . H183 . 106.850 no

H182 . C18 . H183 . 106.922 no

Mn1 . C19. O19 . 178.7(4) yes

Mn1.C20 . O20 . 178.2(4) yes

Mn1.C21.O21.177.5(4) yes

C18.O1.C4 . 119.0(4) yes

C8.S1.C11.91.8(2) yes 


\section{CIF FILE OF 4a}

data_CRYSTALS_cif

_audit_creation_date 05-07-06

_audit_creation_method CRYSTALS_ver_12.24

_oxford_structure_analysis_title '4271055 Compound X'

_chemical_name_systematic

_chemical_melting_point

?
_cell_length_a
_cell_length_b
$9.4006(15)$
$10.4439(15)$
_cell_length_c$$
11.9184(14)
$$$$
102.274(12)
$$$$
104.203(11)
$$$$
112.428(14)
$$
_cell_angle_gamma
_cell_volume
$985.5(3)$
_symmetry_cell_setting
'Triclinic'
_symmetry_space_group_name_H-M 'P -1 '
_symmetry_space_group_name_Hall '?'
loop_




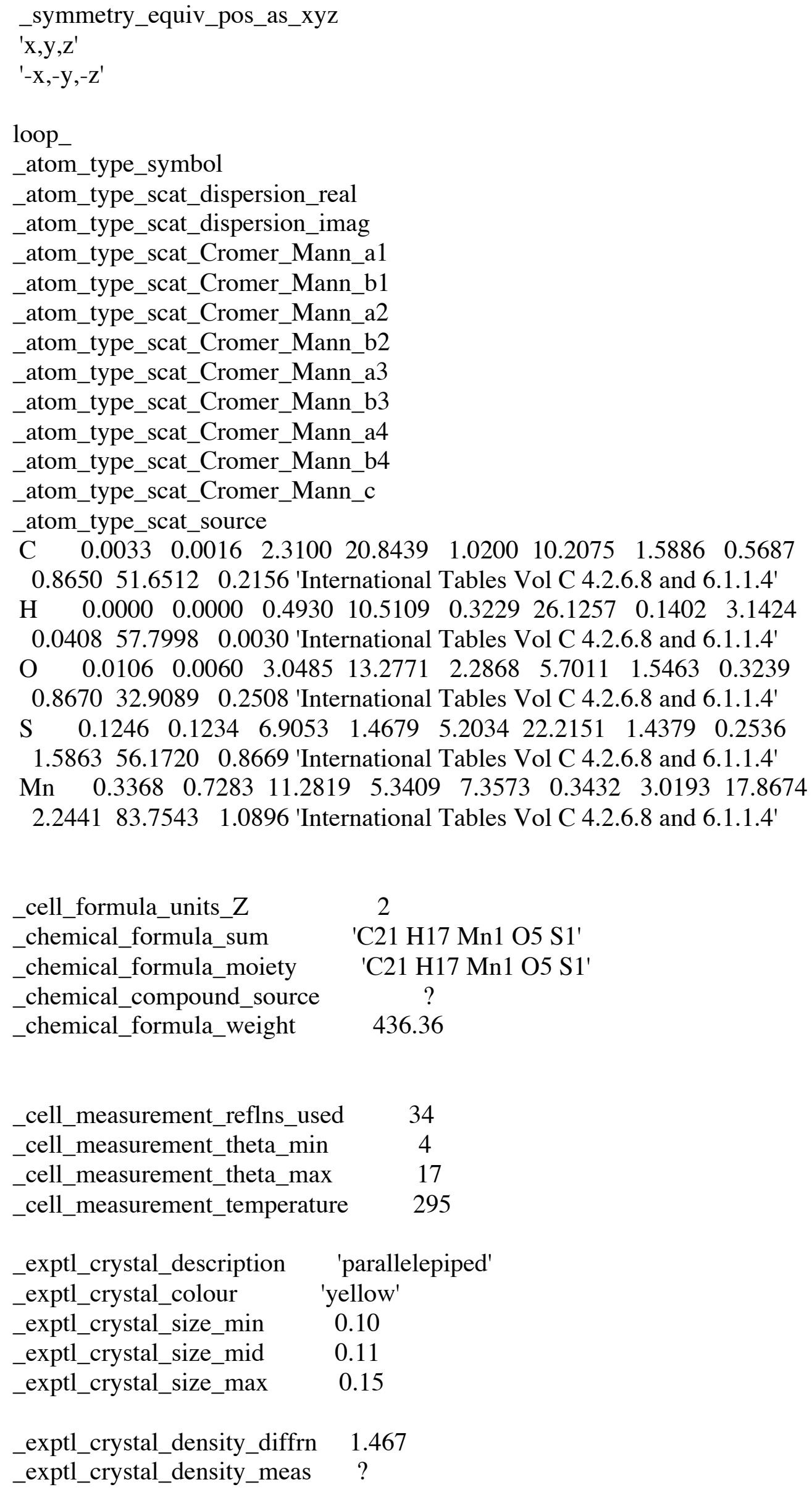


\# Non-dispersive $\mathrm{F}(000)$ :

_exptl_crystal_F_000 448

_exptl_absorpt_coefficient_mu 0.805

_exptl_absorpt_correction_type refdelf

_exptl_absorpt_process_details 'DIFABS (Walker \& Stuart, 1983)'

_exptl_absorpt_correction_T_min 0.86

_exptl_absorpt_correction_T_max 0.92

\# Sheldrick geometric definitions 0.920 .92

_diffrn_measurement_device_type 'Nonius Kappa CCD'

_diffrn_radiation_monochromator 'graphite'

_diffrn_radiation_type_ 'Mo Kla'

_diffrn_radiation_wavelength $\quad 0.71073$

_diffrn_measurement_method Iw

_computing_data_collection 'COLLECT (Nonius BV, 1997)'

_computing_cell_refinement 'Dirax/lsq (Duisenberg \& Schreurs, 1989-2000)'

_computing_data_reduction 'EvalCCD (Duisenberg \& Schreurs 1990-2000)'

_computing_structure_solution 'SHELXS 86 (Sheldrick, 1986)'

_computing_structure_refinement 'CRYSTALS (Betteridge et al 2003)'

_computing_publication_material 'CRYSTALS (Betteridge et al 2003)'

_computing_molecular_graphics 'CAMERON (Watkin et al 1996)'

_diffrn_standards_interval_time?

_diffrn_standards_interval_count ?

_diffrn_standards_number 0

_diffrn_standards_decay_\% ?

_diffrn_ambient_temperature 295

_diffrn_reflns_number 7835

_reflns_number_total 1920

_diffrn_reflns_av_R_equivalents 0.08

\# Number of reflections with Friedels Law is 1920

\# Number of reflections without Friedels Law is 0

\# Theoretical number of reflections is about 3464

_diffrn_reflns_theta_min 2.245

_diffrn_reflns_theta_max 24.996

_diffrn_measured_fraction_theta_max 0.997

_diffrn_reflns_theta_full 24.996

_diffrn_measured_fraction_theta_full 0.997

$\begin{array}{lc}\text { _diffrn_reflns_limit_h_min } & -11 \\ \text { _diffrn_reflns_limit_h_max } & 11 \\ \text { _diffrn_reflns_limit_k_min } & -12 \\ \text { _diffrn_reflns_limit_k_max } & 12\end{array}$




$\begin{array}{lc}\text { _diffrn_reflns_limit_1_min } & -13 \\ \text { _diffrn_reflns_limit_1_max } & 14 \\ \text { _reflns_limit_h_min } & -11 \\ \text { _reflns_limit_h_max } & 10 \\ \text { _reflns_limit_k_min } & -12 \\ \text { _reflns_limit_k_max } & 12 \\ \text { _reflns_limit_l_min } & 0 \\ \text { _reflns_limit_l_max } & 14\end{array}$

_atom_sites_solution_primary direct \#heavy,direct,difmap,geom \#_atom_sites_solution_secondary difmap

_atom_sites_solution_hydrogens geom

_refine_diff_density_min $\quad-0.53$

_refine_diff_density_max $\quad 0.52$

_refine_ls_number_reflns 1920

_refine_ls_number_restraints 0

_refine_ls_number_parameters 254

\#_refine_ls_R_factor_ref 0.0540

_refine_ls_wR_factor_ref 0.0597

_refine_ls_goodness_of_fit_ref 0.9889

\#_reflns_number_all 3446

_refine_ls_R_factor_all 0.1043

_refine_ls_wR_factor_all 0.1316

\# The $\mathrm{I} / \mathrm{u}(\mathrm{I})$ cutoff below was used for refinement as

\# well as the _gt R-factors:

_reflns_threshold_expression I $>1.50 \mathrm{u}(\mathrm{I})$

_reflns_number_gt $\quad 1920$

_refine_ls_R_factor_gt $\quad 0.0540$

_refine_ls_wR_factor_gt 0.0597

_refine_ls_shift/su_max $\quad 0.000259$

\# choose from: rm (reference molecule of known chirality), \# ad (anomolous dispersion - Flack), rmad (rm and ad),

\# syn (from synthesis), unk (unknown) or . (not applicable).

_chemical_absolute_configuration '.'

_refine_ls_structure_factor_coef $\mathrm{F}$

_refine_ls_matrix_type full

_refine_ls_hydrogen_treatment refU \# none, undef, noref, refall, \# refxyz, refU, constr or mixed

_refine_ls_weighting_scheme calc 
_refine_ls_weighting_details

Method, part 1, Chebychev polynomial, (Watkin, 1994, Prince, 1982)

[weight] $\left.=1.0 /[\mathrm{A} \sim 0 \sim * \mathrm{~T} \sim 0 \sim(\mathrm{x})+\mathrm{A} \sim 1 \sim * \mathrm{~T} \sim 1 \sim(\mathrm{x}) . . .+\mathrm{A} \sim \mathrm{n}-1 \sim]^{*} \mathrm{~T} \sim \mathrm{n}-1 \sim(\mathrm{x})\right]$

where $\mathrm{A} \sim \mathrm{i} \sim$ are the Chebychev coefficients listed below and $\mathrm{x}=$ Fcalc/Fmax

Method $=$ Robust Weighting (Prince, 1982)

$\mathrm{W}=[\text { weight }]^{*}\left[1-\left(\mathrm{deltaF} / 6^{*} \operatorname{sigmaF}\right)^{\wedge} 2^{\wedge}\right]^{\wedge} 2^{\wedge}$

$\mathrm{A} \sim \mathrm{i} \sim$ are:

$0.2480 .2110 .925 \mathrm{E}-01$

\# Check this file using the IUCr facility at:

\# http://journals.iucr.org/services/cif/checking/checkfull.html

\# The content below is held in the file 'script/refcif.dat'. This is a text

\# file which you may edit to reflect local conditions.

\# Items which need looking at are represented by a '?'.

\# Items for which there are choices are prefixed with 'choose from'.

_publ_contact_letter

; Please consider this CIF submission for

publication as a Short Format Paper in Acta

Crystallographica $\mathrm{C}$. The figures have been

sent by mail.

_publ_contact_author_name_ 'David Watkin'

_publ_contact_author_address

; Chemical Crystallography Laboratory,

Department of Chemistry,

University of Oxford,

Chemistry Research Laboratory,

Mansfield Road,

Oxford, OX1 3TA, UK.

;

_publ_contact_author_phone '+44 1865 285024'

_publ_contact_author_fax '+44 1865 285018'

_publ_contact_author_email_david.watkin@chem.ox.ac.uk'

_publ_requested_journal_'Organometallics'

_publ_requested_category CO \# choose from: FI FM FO CI CM CO AD

_publ_requested_coeditor_name 'Prof William Clegg'

_publ_section_title \# Title of paper

; ?

;

\# The loop structure below should contain the names and addresses of all \# authors, in the required order of publication. Repeat as necessary. 
loop_

_publ_author_name

_publ_author_address

_publ_author_footnote

'Other, Anthony N.' \# Author 1

; ? \# Address for author 1

;

; ? \# Footnote for author 1

;

'Else, S. O.' \# Author 2

; ? \# Address 2

;

; ? \# Footnote 2

_publ_section_abstract \# Text of the abstract

; ?

_publ_section_comment \# Text of the paper

;?

_publ_section_acknowledgements \# Acknowledgments

; ?

_publ_section_figure_captions \# Captions to figures

; ?

;

_publ_section_exptl_refinement \# see also_refine_ls_hydrogen

; $\mathrm{H}$ atoms placed geometrically after each cycle

;

_publ_section_exptl_prep

; ?

;

\# PROCESSING SUMMARY (IUCr Office Use Only):

_journal_date_recd_electronic ?

_journal_date_to_coeditor?

journal_date_from_coeditor?

_journal_date_accepted ?

journal_date_printers_first ?

journal_date_printers_final?

journal_date_proofs_out?

journal_date_proofs_in?

_journal_coeditor_name?

journal_coeditor_code?

_journal_coeditor_notes?

_journal_techeditor_code?

_journal_techeditor_notes ? 


$\begin{array}{lll}\text { _journal_coden_ASTM } & & ? \\ \text { journal_name_full } & ? & \\ \text { journal_year } & ? & \\ \text { journal_volume } & ? & \\ \text { journal_issue } & ? & \\ \text { journal_page_first } & ? & \\ \text { journal_page_last } & ? \\ \text {-journal_suppl_publ_number } & ? \\ \text { journal_suppl_publ_pages } & ? \\ \text { \# End of 'script/refcif.dat' } & & \end{array}$

\# Insert your own references if required - in alphabetical order _publ_section_references

;

Betteridge, P.W., Carruthers, J.R., Cooper, R.I.,

Prout, K., Watkin, D.J. (2003). J. Appl. Cryst. 36, 1487.

Nonius BV, COLLECT Software, (1997-2001)

Otwinowski, Z. \& Minor, W. (1996), Processing of X-ray

Diffraction Data Collected in Oscillation Mode. Methods Enzymol.

276, 1997, 307-326. Ed Carter, C.W. \& Sweet, R.M., Academic Press.

Prince, E.

Mathematical Techniques in Crystallography

and Materials Science

Springer-Verlag, New York, 1982.

Sheldrick, G.M. (1986). SHELXS86. Program for the solution of crystal structures. Univ. of Gottingen, Federal Republic of Germany.

Walker, N., and Stuart, D. Acta Cryst, (1983) A39, 158-166

Watkin D.J. (1994),

Acta Cryst, A50, 411-437

Watkin, D.J., Prout, C.K. \& Pearce, L.J. (1996) CAMERON, Chemical Crystallography Laboratory, OXFORD, UK.

;

\# Uequiv $=$ arithmetic mean of Ui i.e. Ueqiv $=(\mathrm{U} 1+\mathrm{U} 2+\mathrm{U} 3) / 3$

\# Replace last . with number of unfound hydrogen atoms attached to an atom.

\# ..._refinement_flags_...

\# . no refinement constraints $\quad \mathrm{S}$ special position constraint on site

\# $\mathrm{G}$ rigid group refinement of site $\mathrm{R}$ riding atom 
\# D distance or angle restraint on site $\mathrm{T}$ thermal displacement constraints \# U Uiso or Uij restraint (rigid bond) P partial occupancy constraint

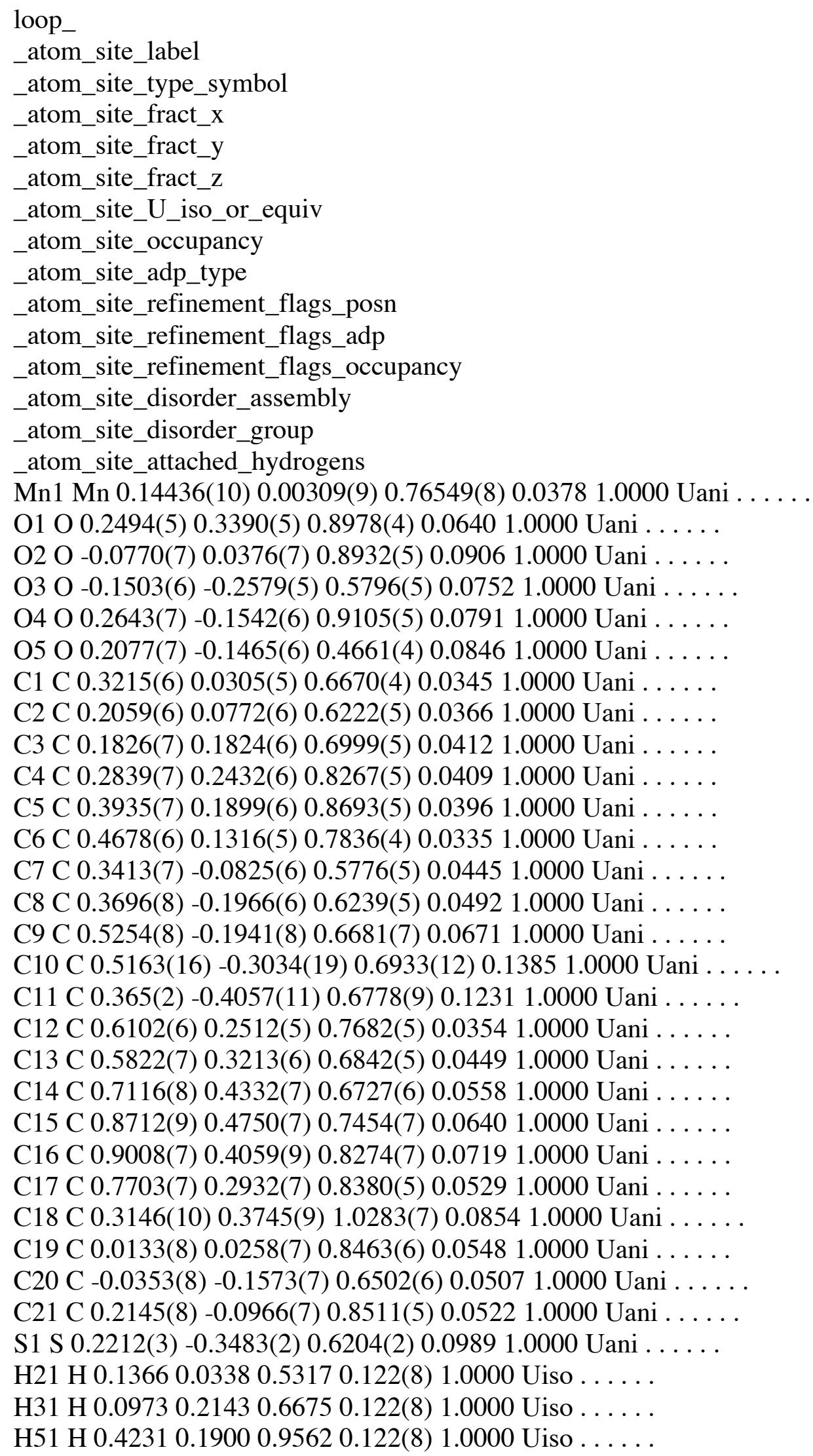


H61 H $0.52630 .07720 .81710 .122(8) 1.0000$ Uiso . . . . . . H71 H $0.4483-0.02600 .5667$ 0.122(8) 1.0000 Uiso . . . . . . H91 H $0.6324-0.10870 .67730 .122(8) 1.0000$ Uiso . . . . . . H101 H $0.6223-0.31700 .72080 .122(8) 1.0000$ Uiso . . . . . . H111 H $0.3443-0.49820 .69690 .122$ (8) 1.0000 Uiso . . . . . . H131 H $0.46440 .28990 .63090 .122(8) 1.0000$ Uiso . . . . . . H141 H $0.68910 .48390 .61170 .122(8) 1.0000$ Uiso . . . . . . H151 H $0.96800 .55670 .73940 .122(8) 1.0000$ Uiso . . . . . . H161 H $1.01840 .43620 .88180 .122(8) 1.0000$ Uiso . . . . . . H171 H $0.79350 .24090 .89750 .122(8) 1.0000$ Uiso . . . . . . H181 H $0.28250 .44431 .07290 .122(8) 1.0000$ Uiso . . . . . . H182 H 0.27210 .28061 .0503 0.122(8) 1.0000 Uiso . . . . . . H183 H 0.43920 .41831 .0598 0.122(8) 1.0000 Uiso . . . . . . loop _atom_site_aniso_label _atom_site_aniso_U_11 _atom_site_aniso_U_22 _atom_site_aniso_U_33 _atom_site_aniso_U_23 _atom_site_aniso_U_13 _atom_site_aniso_U_12

Mn1 0.0370(5) 0.0395(5) 0.0368(5) 0.0142(4) 0.0130(4) 0.0170(4) O1 0.070(3) 0.052(3) 0.065(3) $-0.001(2) 0.023(2) 0.035(2)$ O2 0.088(4) 0.126(5) 0.098(4) 0.047(4) 0.064(4) 0.064(4) O3 0.058(3) 0.054(3) 0.073(3) $0.015(3) 0.007(3) 0.000(3)$ O4 0.099(4) $0.085(4) 0.074(3) 0.053(3) 0.029(3) 0.050(3)$ O5 0.120(4) 0.090(4) 0.038(2) -0.002(2) -0.003(3) 0.074(3) C1 0.039(3) 0.033(3) 0.028(3) 0.011(2) 0.012(2) 0.014(2) C2 0.039(3) 0.040(3) 0.031(3) 0.017(2) 0.008(2) 0.019(3) C3 0.043(3) 0.042(3) 0.048(3) 0.022(3) 0.016(3) 0.025(3) C4 0.046(3) 0.032(3) 0.040(3) 0.008(3) 0.012(3) 0.018(3) C5 0.041(3) 0.038(3) 0.030(3) 0.007(2) 0.007(2) 0.015(3) C6 0.036(3) 0.032(3) 0.030(3) 0.010(2) 0.007(2) 0.017(2) C7 0.049(3) 0.040(3) 0.038(3) 0.005(3) 0.015(3) 0.019(3) C8 0.066(4) 0.047(3) 0.043(3) 0.012(3) 0.020(3) 0.036(3) C9 0.059(4) 0.055(4) 0.072(5) -0.006(4) $-0.011(4) 0.047(4)$ C10 0.121(10) 0.160(13) 0.118(9) 0.032(9) 0.017(8) 0.072(10) C11 0.257(15) 0.081(7) 0.104(7) 0.057(6) 0.078(9) 0.126(9) C12 0.037(3) 0.031(3) 0.032(3) 0.001(2) 0.010(2) 0.015(2) C13 0.051(4) 0.038(3) 0.043(3) 0.012(3) 0.016(3) 0.019(3) C14 0.060(4) 0.040(4) 0.061(4) 0.016(3) 0.023(4) 0.018(3) C15 0.065(5) 0.049(4) 0.066(4) 0.010(4) 0.036(4) 0.012(4) C16 0.029(4) 0.086(5) 0.068(5) 0.003(4) 0.011(3) 0.010(4) C17 0.038(4) 0.067(4) 0.049(4) 0.016(3) 0.010(3) 0.025(3) C18 0.092(6) 0.084(6) 0.061(5) -0.012(4) 0.030(4) 0.039(5) C19 0.054(4) 0.058(4) 0.052(4) 0.022(3) 0.020(3) 0.023(3) C20 0.044(4) 0.052(4) 0.058(4) 0.025(4) 0.023(3) 0.018(3) C21 0.058(4) 0.054(4) 0.046(4) 0.025(3) 0.022(3) 0.022(3) S1 0.1155(19) 0.0591(13) 0.123(2) 0.0310(13) 0.0671(16) 0.0268(13) 
_refine_ls_extinction_method 'None'

loop_

_geom_bond_atom_site_label_1

_geom_bond_site_symmetry_1

_geom_bond_atom_site_label_2

_geom_bond_site_symmetry_2

_geom_bond_distance

_geom_bond_publ_flag

Mn1 . C1 . 2.230(5) yes

Mn1.C2 . 2.135(5) yes

Mn1.C3 . 2.120(5) yes

Mn1.C4.2.188(5) yes

Mn1.C5 . 2.211(5) yes

Mn1 . C19.1.792(7) yes

Mn1.C20.1.808(7) yes

Mn1.C21.1.790(6) yes

O1.C4.1.359(6) yes

O1 . C18.1.430(9) yes

$\mathrm{O} 2 . \mathrm{C} 19.1 .153(7)$ yes

O3. C20.1.137(7) yes

O4.C21.1.147(7) yes

O5.C7 . 1.398(7) yes

C1.C2.1.390(7) yes

C1.C6. 1.506(7) yes

C1.C7. 1.514(7) yes

C2.C3.1.402(7) yes

$\mathrm{C} 2 . \mathrm{H} 21.1 .007$ no

C3 . C4 . 1.420(8) yes

C3 . H31. 1.005 no

C4.C5 . 1.390(8) yes

C5 . C6 . 1.521(7) yes

C5 . H51. 1.004 no

C6. C12. 1.526(7) yes

C6. H61. 1.005 no

C7.C8.1.498(8) yes

C7 . H71 . 1.010 no

C8.C9.1.417(8) yes

C8.S1 . 1.648(7) yes

C9 . C10 . 1.218(16) yes

C9. H91 . 1.020 no

C10.C11.1.348(17) yes

C10. H101. 1.043 no

C11.S1.1.734(12) yes

C11. H111. 0.997 no

C12.C13 . 1.392(7) yes

C12.C17. 1.370(8) yes

C13.C14.1.389(8) yes

C13. H131. 1.013 no

C14.C15 . 1.376(9) yes 
C14 . H141 . 1.013 no

C15 . C16 . 1.368(10) yes

C15. H151 . 1.013 no

C16.C17 . 1.394(9) yes

C16 . H161 . 1.018 no

C17.H171. 1.015 no

C18. H181. 0.993 no

C18. H182 . 1.025 no

C18. H183 . 1.012 no

loop_

_geom_angle_atom_site_label_1

_geom_angle_site_symmetry_1

_geom_angle_atom_site_label_2

_geom_angle_site_symmetry_2

_geom_angle_atom_site_label_3

_geom_angle_site_symmetry_3

_geom_angle

_geom_angle_publ_flag

C1 . Mn1 . C2 . 37.07(18) yes

C1 . Mn1 . C3 . 68.11(19) yes

C2 . Mn1 . C3 . 38.5(2) yes

C1. Mn1 . C4 . 78.51(19) yes

C2. Mn1.C4 .68.0(2) yes

C3 . Mn1.C4 . 38.5(2) yes

C1. Mn1 . C5 . 64.88(18) yes

C2 . Mn1 . C5 . 78.50(19) yes

C3 . Mn1.C5 . 67.9(2) yes

C4 . Mn1 . C5 . 36.8(2) yes

C1 . Mn1 . C19 . 166.1(2) yes

C2 . Mn1 . C19. 132.6(2) yes

C3 . Mn1 . C19 . 98.6(2) yes

C4. Mn1 . C19. 88.5(2) yes

C5 . Mn1 . C19 . 107.2(2) yes

C1. Mn1 . C20.99.9(2) yes

C2 . Mn1 . C20 . 89.3(2) yes

C3 . Mn1 . C20 . 107.7(2) yes

C4 . Mn1 . C20 . 144.7(2) yes

C5 . Mn1.C20. 164.7(2) yes

C1 . Mn1 . C21 . 95.1(2) yes

C2 . Mn1 . C21 . 131.8(2) yes

C3 . Mn1 . C21 . 153.0(2) yes

C4 . Mn1.C21 . 119.7(2) yes

C5 . Mn1 . C21.86.0(2) yes

C19. Mn1 . C20 . 87.8(3) yes

C19. Mn1.C21.95.6(3) yes

C20.Mn1.C21.95.6(3) yes

C4.O1.C18.117.6(5) yes

Mn1.C1.C2 . 67.7(3) yes

Mn1.C1.C6 . 92.5(3) yes

C2 . C1 . C6 . 119.0(4) yes 
Mn1.C1 . C7 . 130.9(4) yes

C2 . C1 . C7 . 118.9(4) yes

C6.C1.C7.117.2(4) yes

Mn1.C2 . C1 . 75.2(3) yes

Mn1.C2 . C3 . 70.2(3) yes

C1.C2 .C3 . 121.6(5) yes

Mn1.C2 . H21 . 127.239 no

C1 . C2 . H21 . 119.210 no

C3 . C2 . H21 . 119.169 no

Mn1.C3 . C2 . 71.3(3) yes

Mn1.C3 . C4 .73.4(3) yes

C2 . C3.C4 . 117.9(5) yes

Mn1.C3 . H31 . 126.073 no

C2 . C3 . H31 . 121.382 no

C4 . C3 . H31 . 120.750 no

Mn1.C4.C3 .68.2(3) yes

Mn1.C4.O1. 124.6(4) yes

C3.C4.O1.114.8(5) yes

Mn1.C4.C5 .72.5(3) yes

C3.C4 . C5 . 119.0(5) yes

O1.C4.C5 . 125.8(5) yes

Mn1 . C5 . C4 .70.7(3) yes

Mn1.C5 .C6. 92.9(3) yes

C4.C5.C6 . 119.6(4) yes

Mn1 . C5 . H51 . 106.130 no

C4 . C5 . H51 . 119.941 no

C6.C5.H51. 120.432 no

C5 . C6.C1. 103.8(4) yes

C5 . C6 . C12.114.3(4) yes

C1 . C6 . C12 . 115.5(4) yes

C5 . C6 . H61 . 113.044 no

C1 . C6 . H61 . 111.087 no

C12.C6. H61 . 99.512 no

C1.C7.O5 . 109.3(5) yes

C1.C7.C8.115.8(4) yes

O5 . C7 . C8 . 111.8(5) yes

C1.C7 . H71 . 105.509 no

O5.C7.H71.111.125 no

C8 . C7 . H71 . 103.029 no

C7 . C8 . C9 . 124.7(6) yes

C7.C8.S1.124.2(5) yes

C9.C8.S1 . 110.9(5) yes

C8 . C9 . C10 . 112.9(9) yes

C8 . C9 . H91 . 122.466 no

C10 . C9 . H91 . 124.638 no

C9.C10.C11.117.4(12) yes

C9 . C10 . H101 . 119.603 no

C11.C10 . H101. 122.888 no

C10 . C11 . S1 . 108.7(8) yes

C10 . C11 . H111 . 124.029 no 
S1 . C11.H111 . 127.282 no C6. C12 . C13 . 121.5(5) yes C6.C12.C17. 120.5(5) yes

C13 . C12 . C17 . 118.1(5) yes C12.C13 . C14.121.5(5) yes C12.C13 . H131 . 118.795 no C14.C13 . H131 . 119.709 no C13 . C14 . C15 . 119.2(6) yes C13 . C14 . H141 . 120.542 no C15 . C14 . H141 . 120.223 no C14.C15 . C16. 119.9(6) yes C14.C15 . H151 . 120.935 no C16 . C15 . H151 . 119.128 no C15 . C16.C17 . 120.5(6) yes C15 . C16 . H161 . 120.551 no C17.C16. H161 . 118.949 no C16.C17 . C12 . 120.7(6) yes C16 . C17 . H171 . 119.966 no C12.C17 . H171 . 119.291 no O1 . C18 . H181 . 112.167 no O1 . C18 . H182 . 110.061 no H181 . C18 . H182 . 108.005 no O1 . C18 . H183 . 110.844 no H181 . C18 . H183 . 109.040 no H182 . C18 . H183 . 106.523 no O2 . C19. Mn1 . 176.9(6) yes Mn1.C20.O3 . 178.4(5) yes O4 . C21. Mn1 . 176.7(6) yes C11.S1.C8.90.1(5) yes 


\section{. Crystal structure analysis of $\mathbf{3 a}$}

Crystal structure data are summarised in Table 1. Data were recorded at room temperature on a Kappa-CCD Enraf-Nonius diffractometer with graphite mono-chromated $\mathrm{Mo}_{\mathrm{K} \alpha}$ radiation $(\lambda=$ $0.71073 \AA$ ) and the $\omega$-scan technique. Orientation matrix and lattice parameters were obtained by least-squares refinement of the diffraction data of 80 reflections within the range of $4^{\circ}<\theta<21^{\circ}$. The index ranges of data collection were $-10 \leq \mathrm{h} \leq 12,-24 \leq \mathrm{k} \leq 22,-13 \leq 1 \leq 14$. Intensity data were collected in the $\theta$ range $2.0-27.5^{\circ}, 2124$ have $(\mathrm{Fo})^{2}-3 \sigma(\mathrm{Fo})^{2}$. All the measured independent reflections were used in the analysis. The structure was solved by direct methods and refined with fullmatrix least-squares technique on $F$ using the CRYSTALS ${ }^{1}$ programs. All non-hydrogen atoms were refined anisotropically. All hydrogen atoms were either set in calculated positions and isotropically refined. The values of the discrepancy indices $R 1\left(R_{\mathrm{w} 2}\right)$ for all data were $0.1058(0.0451)$, whereas those listed in Table 1 correspond to the data with $I>3 \sigma(I)$. The final Fourier-difference map showed maximum and minimum height peaks of 0.383 and $-0.340 \mathrm{e} \AA^{-3}$. The values of number of reflections/number of variable parameters are 254, and those of the goodness-of-fit are 1.136. Molecular structure was drawn with the program CAMERON and is reported in Figure 1.

(1) Betteridge, P. W., Carruthers, J. R., Cooper, R. I., Prout, K., Watkin, D. J. (2003). J. Appl. Cryst. $36,1487$.

(2) Watkin, D. J., Prout, C. K. \& Pearce, L. J. (1996), CAMERON, Chemical Crystallography Laboratory, OXFORD, UK

\section{TABLE I CRYSTAL DATA OF 3a}

\begin{tabular}{|c|c|}
\hline Formula & $\mathrm{C}_{21} \mathrm{H}_{15} \mathrm{O}_{5} \mathrm{MnS}$ \\
\hline Fw $=$ & 434.34 \\
\hline Crystal System & Monoclinic \\
\hline $\mathbf{a}(\AA)=$ & $9.4929(6)$ \\
\hline b $(\underset{0}{\AA})=$ & $11.3400(8)$ \\
\hline c $(\AA)=$ & $19.1506(12)$ \\
\hline$\alpha\left(^{\circ}\right)=$ & 90 \\
\hline$\beta\left({ }^{\circ}\right)=$ & $100.134(4)$ \\
\hline$\gamma\left(\left(^{\circ}\right)=\right.$ & 90 \\
\hline $\mathbf{V}\left(\AA^{3}\right)=$ & $2029.4(3)$ \\
\hline $\mathrm{Z}=$ & 4 \\
\hline Space group & $\mathbf{P} 2 \mathbf{2}_{1} / \mathbf{n}$ \\
\hline Crystal shape & Parallelepiped \\
\hline Crystal colour & yellow \\
\hline Linear absorption coefficient $\mu\left(\mathrm{cm}^{-1}\right)$ & 7.816 \\
\hline Density $\rho\left(\mathrm{g} \mathrm{cm}^{3}\right)$ & 1.42 \\
\hline
\end{tabular}

\section{Diffractometer}

$\mathrm{C}_{21} \mathrm{H}_{15} \mathrm{O}_{5} \mathrm{MnS}$

434.34

Monoclinic

$9.4929(6)$

$11.3400(8)$

90

90

$2029.4(3)$

P 2 1 /n

Parallelepiped

KAPPACCD-Enraf Nonius 
Radiation

$\theta$ Limits $\left(^{\circ}\right)$

Octants collected

$\mathrm{Nb}$ of data collected

$\mathrm{Nb}$ of unique data collected

$\mathrm{Nb}$ of unique data used for refinement

Merging R

$\mathbf{R}=\Sigma||$ Fo $|-|$ Fc ||$/ \Sigma \mid$ Fol

$\mathbf{R w}^{*}=\left[\Sigma \mathbf{w}(\|\mathrm{Fol}-\mid \mathrm{Fc}\|)^{2} / \Sigma \mathbf{w F o}^{2}\right]^{1 / 2}$

Absorption correction

Secondary extinction coefficient

Goodness of fit

$\mathrm{Nb}$ of variables

$\Delta \rho \min \left(\mathrm{e}^{\mathrm{a}} \AA^{3}\right)$

$\Delta \rho \max \left(\mathbf{e} / \AA^{3}\right)$
$\operatorname{MoK} \alpha(\lambda=0.71073 \AA)$

2-27.5

$-10,12 ;-24,22 ;-13,14$

12729

4629

$2124(\text { Fo })^{2}>3 \sigma(\text { Fo })^{2}$

0.036

0.0392

0.0433

sadabs

none

1.136

254

$-0.340$

0.383

* Weighting scheme of the form $w=w^{\prime}\left[1-(\text { ( I Fo I - | Fc || ) / } 6 \sigma(\mathrm{Fo}))^{2}\right]^{2}$ with $w^{\prime}=1 / \Sigma_{\mathbf{r}}$ $A_{r} T_{r}(X)$ with coefficients $2.61,-2.38,2.55,-0.834$ and 0.468 for a Chebyshev serie for which $\mathrm{X}=\mathrm{Fc} / \mathrm{Fc}$ (max) 
Table II : Fractional atomic coordinates for $\mathrm{C}_{21} \mathrm{H}_{15} \mathrm{O}_{5} \mathrm{MnS}$

\begin{tabular}{|c|c|c|c|c|}
\hline Atom & $x / a$ & $\mathrm{y} / \mathrm{b}$ & $z / c$ & $\mathrm{U}$ (eqv) \\
\hline $\operatorname{Mn}(1)$ & $0.12307(6)$ & $0.05321(5)$ & $0.86314(3)$ & 0.0421 \\
\hline$C(1)$ & $0.1399(4)$ & $0.0012(3)$ & $0.75442(18)$ & 0.0361 \\
\hline$C(2)$ & $0.0644(4)$ & $-0.0839(3)$ & $0.78858(19)$ & 0.0430 \\
\hline$C(3)$ & $-0.0571(4)$ & $-0.0527(4)$ & $0.81613(19)$ & 0.0487 \\
\hline$C(4)$ & $-0.1033(4)$ & $0.0667(4)$ & $0.8102(2)$ & 0.0481 \\
\hline$C(5)$ & $-0.0247(4)$ & 0.1489 (3) & $0.7797(2)$ & 0.0435 \\
\hline$C(6)$ & $0.0596(3)$ & $0.1102(3)$ & $0.72300(17)$ & 0.0333 \\
\hline$C(7)$ & $0.2827(4)$ & $-0.0349(3)$ & $0.74212(18)$ & 0.0404 \\
\hline$C(8)$ & $0.3592(4)$ & $0.0312(3)$ & $0.6934(2)$ & 0.0411 \\
\hline$C(9)$ & $0.3177(4)$ & $0.1106(4)$ & $0.6393(2)$ & 0.0457 \\
\hline$C(10)$ & $0.4311(5)$ & $0.1455(4)$ & $0.6053(3)$ & 0.0641 \\
\hline$C(11)$ & $0.5549(6)$ & $0.0938(5)$ & $0.6334(4)$ & 0.0870 \\
\hline$C(12)$ & $-0.0313(3)$ & $0.0978(3)$ & $0.64900(18)$ & 0.0365 \\
\hline$C(13)$ & $-0.0692(4)$ & $-0.0097(4)$ & $0.6173(2)$ & 0.0507 \\
\hline$C(14)$ & $-0.1486(5)$ & $-0.0141(4)$ & $0.5487(2)$ & 0.0618 \\
\hline$C(15)$ & $-0.1894(5)$ & $0.0876(5)$ & $0.5122(2)$ & 0.0671 \\
\hline$C(16)$ & $-0.1513(5)$ & $0.1940(5)$ & $0.5432(3)$ & 0.0671 \\
\hline$C(17)$ & $-0.0727(4)$ & $0.2005(4)$ & $0.6116(2)$ & 0.0544 \\
\hline$C(18)$ & $-0.2603(7)$ & $0.2077(5)$ & $0.8468(4)$ & 0.1051 \\
\hline$C(19)$ & $0.0664(5)$ & $0.1055(4)$ & $0.9428(2)$ & 0.0596 \\
\hline$C(20)$ & $0.2460(5)$ & $-0.0465(4)$ & $0.9169(2)$ & 0.0617 \\
\hline$C(21)$ & $0.2571(5)$ & $0.1655(4)$ & $0.8619(2)$ & 0.0496 \\
\hline$O(1)$ & $-0.2122(3)$ & 0.0915 (3) & $0.84541(18)$ & 0.0706 \\
\hline$O(5)$ & $0.3442(3)$ & $-0.1202(3)$ & $0.77379(15)$ & 0.0590 \\
\hline$O(19)$ & $0.0310(4)$ & $0.1408(4)$ & $0.99291(18)$ & 0.0921 \\
\hline$O(20)$ & $0.3221(5)$ & $-0.1084(4)$ & $0.9523(2)$ & 0.1037 \\
\hline$O(21)$ & $0.3436(4)$ & $0.2348(3)$ & $0.85905(17)$ & 0.0770 \\
\hline$s(1)$ & $0.53844(13)$ & $-0.00049(14)$ & $0.70044(10)$ & 0.0906 \\
\hline
\end{tabular}


Table III : Interatomic distances (A) for $\mathrm{C}_{21} \mathrm{H}_{15} \mathrm{O}_{5} \mathrm{MnS}$

$\begin{array}{llll}M n(1)-C(1) & 2.197(3) & M n(1)-C(2) & 2.119(3) \\ M n(1)-C(3) & 2.153(4) & M n(1)-C(4) & 2.214(4) \\ M n(1)-C(5) & 2.215(4) & M n(1)-C(19) & 1.805(4) \\ M n(1)-C(20) & 1.810(5) & M n(1)-C(21) & 1.804(4) \\ C(1)-C(2) & 1.428(5) & C(1)-C(6) & 1.519(5) \\ C(1)-C(7) & 1.474(5) & C(2)-C(3) & 1.396(5) \\ C(3)-C(4) & 1.422(6) & C(4)-C(5) & 1.385(5) \\ C(4)-O(1) & 1.360(5) & C(5)-C(6) & 1.522(5) \\ C(6)-C(12) & 1.531(5) & C(7)-C(8) & 1.482(5) \\ C(7)-O(5) & 1.233(4) & C(8)-C(9) & 1.377(5) \\ C(8)-S(1) & 1.721(4) & C(9)-C(10) & 1.409(6) \\ C(10)-C(11) & 1.339(7) & C(11)-S(1) & 1.699(6) \\ C(12)-C(13) & 1.381(5) & C(12)-C(17) & 1.387(5) \\ C(13)-C(14) & 1.396(6) & C(14)-C(15) & 1.368(7) \\ C(15)-C(16) & 1.365(7) & C(16)-C(17) & 1.391(6) \\ C(18)-O(1) & 1.396(6) & C(19)-O(19) & 1.143(5) \\ C(20)-O(20) & 1.141(5) & C(21)-O(21) & 1.144(5)\end{array}$

Table IV :Bond angles $\left({ }^{\circ}\right)$ for $\mathrm{C}_{21} \mathrm{H}_{15} \mathrm{O}_{5} \mathrm{MnS}$

$\begin{array}{llrlrr}C(1) & -M n(1)-C(2) & 38.59(14) & C(1)-M n(1)-C(3) & 68.83(13) \\ C(2)-M n(1)-C(3) & 38.15(15) & C(1)-M n(1)-C(4) & 79.11(13) \\ C(2)-M n(1)-C(4) & 67.87(15) & C(3)-M n(1)-C(4) & 37.96(15) \\ C(1)-M n(1)-C(5) & 65.81(13) & C(2)-M n(1)-C(5) & 79.36(14) \\ C(3)-M n(1)-C(5) & 67.42(15) & C(4)-M n(1)-C(5) & 36.45(14) \\ C(1)-M n(1)-C(19) & 166.33(18) & C(2)-M n(1)-C(19) & 136.79(18) \\ C(3)-M n(1)-C(19) & 101.75(17) & C(4)-M n(1)-C(19) & 87.42(18) \\ C(5)-M n(1)-C(19) & 101.77(18) & C(1)-M n(1)-C(20) & 102.95(16) \\ C(2)-M n(1)-C(20) & 89.51(17) & C(3)-M n(1)-C(20) & 105.95(19) \\ C(4)-M n(1)-C(20) & 141.30(19) & C(5)-M n(1)-C(20) & 168.20(16) \\ C(1)-M n(1)-C(21) & 90.45(15) & C(2)-M n(1)-C(21) & 127.72(16) \\ C(3)-M n(1)-C(21) & 153.98(16) & C(4)-M n(1)-C(21) & 125.10(17) \\ C(5)-M n(1)-C(21) & 90.18(16) & C(19)-M n(1)-C(20) & 89.0(2) \\ C(19)-M n(1)-C(21) & 95.46(19) & C(20)-M n(1)-C(21) & 93.6(2) \\ M n(1)-C(1) & -C(2) & 67.74(19) & M n(1)-C(1)-C(6) & 92.4(2) \\ C(2)-C(1)-C(6) & 118.3(3) & M n(1)-C(1)-C(7) & 116.6(2) \\ C(2)-C(1)-C(7) & 115.8(3) & C(6)-C(1)-C(7) & 125.0(3) \\ M n(1)-C(2)-C(1) & 73.7(2) & M n(1)-C(2)-C(3) & 72.3(2) \\ C(1)-C(2)-C(3) & 121.1(3) & M n(1)-C(3)-C(2) & 69.6(2) \\ M n(1)-C(3)-C(4) & 73.3(2) & C(2)-C(3)-C(4) & 118.4(3) \\ M n(1)-C(4)-C(3) & 68.7(2) & M n(1)-C(4)-C(5) & 71.8(2) \\ C(3)-C(4)-C(5) & 119.5(3) & M n(1)-C(4)-O(1) & 123.3(3) \\ C(3)-C(4)-O(1) & 114.1(4) & C(5)-C(4)-O(1) & 125.7(4) \\ M n(1)-C(5)-C(4) & 71.7(2) & M n(1)-C(5)-C(6) & 91.6(2) \\ C(4)-C(5)-C(6) & 119.8(3) & C(1)-C(6)-C(5) & 104.0(3) \\ C(1)-C(6)-C(12) & 117.0(3) & C(5)-C(6)-C(12) & 113.9(3) \\ C(1)-C(7)-C(8) & 121.8(3) & C(1)-C(7)-O(5) & 120.6(3) \\ C(8)-C(7)-O(5) & 117.6(3) & C(7)-C(8)-C(9) & 134.2(3) \\ C(7)-C(8)-S(1) & 115.6(3) & C(9)-C(8)-S(1) & 110.1(3) \\ C(8)-C(9)-C(10) & 112.9(4) & C(9)-C(10)-C(11) & 112.3(4) \\ C(10)-C(11)-S(1) & 112.8(4) & C(6)-C(12)-C(13) & 123.3(3) \\ C(6)-C(12)-C(17) & 117.6(3) & C(13)-C(12)-C(17) & 119.1(3) \\ C(12)-C(13)-C(14) & 120.0(4) & C(13)-C(14)-C(15) & 120.5(4) \\ C(14)-C(15)-C(16) & 119.6(4) & C(15)-C(16)-C(17) & 120.8(4) \\ C(12)-C(17)-C(16) & 119.9(4) & M n(1)-C(19)-O(19) & 178.7(4) \\ M n(1)-C(20)-O(20) & 178.2(4) & M n(1)-C(21)-O(21) & 177.5(4) \\ C(4)-O(1)-C(18) & 119.0(4) & C(8)-S(1)-C(11) & 91.8(2)\end{array}$


Table S1 : Anisotropic thermal parameters for $\mathrm{C}_{21} \mathrm{H}_{15} \mathrm{O}_{5} \mathrm{MnS}$

\begin{tabular}{|c|c|c|c|c|c|c|}
\hline Atom & $\mathrm{u}(11)$ & $u(22)$ & $\mathrm{u}(33)$ & $u(23)$ & $\mathrm{u}(13)$ & $u(12)$ \\
\hline $\operatorname{Mn}(1)$ & $0.0504(3)$ & $0.0398(3)$ & $0.0364(3)$ & $-0.0020(3)$ & $0.0086(2)$ & $-0.0058(3)$ \\
\hline$C(1)$ & $0.0416(19)$ & $0.0301(17)$ & $0.0362(17)$ & $-0.0036(15)$ & $0.0053(15)$ & $0.0002(16)$ \\
\hline$C(2)$ & $0.054(2)$ & $0.033(2)$ & 0.0403 (19) & $-0.0032(15)$ & $0.0031(17)$ & $-0.0047(17)$ \\
\hline$C(3)$ & $0.053(2)$ & $0.046(2)$ & $0.048(2)$ & $-0.002(2)$ & $0.0127(18)$ & $-0.015(2)$ \\
\hline$C(4)$ & $0.041(2)$ & $0.055(3)$ & $0.050(2)$ & $-0.009(2)$ & 0.0122 (17) & $-0.0023(19)$ \\
\hline$C(5)$ & $0.045(2)$ & $0.038(2)$ & $0.047(2)$ & $-0.0045(17)$ & $0.0090(17)$ & $0.0000(17)$ \\
\hline$C(7)$ & $0.0418(19)$ & $0.035(2)$ & $0.0419(18)$ & $-0.0099(16)$ & $-0.0007(16)$ & $0.0000(16)$ \\
\hline$C(8)$ & $0.0322(17)$ & $0.035(2)$ & $0.056(2)$ & $-0.0125(16)$ & $0.0065(16)$ & $-0.0011(15)$ \\
\hline$C(9)$ & $0.041(2)$ & $0.052(2)$ & $0.047(2)$ & $-0.0002(19)$ & $0.0143(17)$ & $-0.0029(18)$ \\
\hline$C(10)$ & $0.064(3)$ & $0.062(3)$ & $0.073(3)$ & $-0.009(2)$ & $0.033(2)$ & $-0.007(2)$ \\
\hline$C(11)$ & $0.062(3)$ & $0.077(4)$ & $0.136(5)$ & $-0.005(3)$ & $0.055(3)$ & $-0.014(3)$ \\
\hline$C(12)$ & $0.0285(17)$ & $0.0385(19)$ & $0.0429(19)$ & $0.0057(16)$ & $0.0071(15)$ & $0.0031(15)$ \\
\hline$C(13)$ & $0.046(2)$ & $0.049(2)$ & $0.053(2)$ & $-0.0047(19)$ & $-0.0023(19)$ & $0.0038(19)$ \\
\hline$C(15)$ & $0.059(3)$ & $0.092(4)$ & $0.044(2)$ & $0.005(2)$ & $-0.008(2)$ & $-0.004(3)$ \\
\hline$C(16)$ & $0.058(3)$ & $0.076(3)$ & $0.060(3)$ & $0.023(3)$ & $-0.009(2)$ & $0.000(3)$ \\
\hline$C(17)$ & $0.048(2)$ & $0.051(3)$ & $0.060(2)$ & $0.012(2)$ & $-0.0034(19)$ & $-0.004(2)$ \\
\hline$C(18)$ & $0.088(4)$ & $0.097(5)$ & $0.143(6)$ & $0.001(4)$ & $0.057(4)$ & $0.021(4)$ \\
\hline C (19) & $0.070(3)$ & $0.059(3)$ & $0.052(2)$ & $-0.007(2)$ & $0.016(2)$ & $-0.015(2)$ \\
\hline$C(20)$ & $0.076(3)$ & $0.057(3)$ & $0.048(2)$ & $-0.001(2)$ & $0.001(2)$ & $0.005(3)$ \\
\hline$C(21)$ & $0.053(2)$ & $0.054(3)$ & $0.040(2)$ & $-0.0056(18)$ & $0.0050(18)$ & $-0.007(2)$ \\
\hline$O(1)$ & $0.0571(18)$ & $0.079(2)$ & $0.084(2)$ & $-0.0112(18)$ & $0.0358(17)$ & $0.0007(16)$ \\
\hline$O(5)$ & $0.0622(18)$ & $0.0447(17)$ & $0.0674(18)$ & $0.0087(14)$ & $0.0042(15)$ & $0.0191(14)$ \\
\hline$O(19)$ & $0.123(3)$ & $0.097(3)$ & $0.068(2)$ & $-0.028(2)$ & $0.050(2)$ & $-0.021(2)$ \\
\hline$O(20)$ & $0.120(3)$ & $0.105(3)$ & $0.074(2)$ & $0.022(2)$ & $-0.014(2)$ & $0.032(3)$ \\
\hline$O(21)$ & $0.078(2)$ & $0.079(2)$ & $0.070(2)$ & $-0.0079(18)$ & $0.0036(17)$ & $-0.038(2)$ \\
\hline$S(1)$ & $0.0408(6)$ & $0.0877(10)$ & $0.1452(14)$ & $0.0205(10)$ & $0.0214(7)$ & $0.0134(6)$ \\
\hline
\end{tabular}

$\mathrm{C}_{21} \mathrm{H}_{15} \mathrm{O}_{5} \mathrm{MnS}$

Table S2 : Hydrogen atoms fractional atomic coordinates for

$\begin{array}{lrrrr}\text { Atom } & \mathbf{x} / \mathrm{a} & \mathrm{y} / \mathrm{b} & \mathrm{z} / \mathrm{c} & \mathrm{U}(\mathrm{iso}) \\ \mathrm{H}(21) & & & & \\ \mathrm{H}(31) & 0.1008 & -0.1679 & 0.7934 & 0.082(4) \\ \mathrm{H}(51) & -0.1116 & -0.1131 & 0.8398 & 0.082(4) \\ \mathrm{H}(61) & -0.0246 & 0.2341 & 0.7950 & 0.082(4) \\ \mathrm{H}(91) & 0.1335 & 0.1733 & 0.7198 & 0.082(4) \\ \mathrm{H}(101) & 0.2159 & 0.1403 & 0.6254 & 0.082(4) \\ \mathrm{H}(111) & 0.4201 & 0.2042 & 0.5643 & 0.082(4) \\ \mathrm{H}(131) & 0.6485 & 0.1086 & 0.6163 & 0.082(4) \\ \mathrm{H}(141) & -0.0391 & -0.0856 & 0.6442 & 0.082(4) \\ \mathrm{H}(151) & -0.1770 & -0.0932 & 0.5254 & 0.082(4) \\ \mathrm{H}(161) & -0.2481 & 0.0839 & 0.4626 & 0.082(4) \\ \mathrm{H}(171) & -0.1807 & 0.2692 & 0.5153 & 0.082(4) \\ \mathrm{H}(181) & -0.0458 & 0.2804 & 0.6346 & 0.082(4) \\ \mathrm{H}(182) & -0.3418 & 0.2150 & 0.8737 & 0.082(4) \\ \mathrm{H}(183) & -0.1819 & 0.2630 & 0.8682 & 0.082(4) \\ & -0.2975 & 0.2371 & 0.7961 & 0.082(4)\end{array}$


Crystal structure data of $\mathbf{4 a}$ are summarised in Table 2.

Data were recorded at room temperature on a Kappa-CCD Enraf-Nonius diffractometer with graphite mono-chromated $\mathrm{Mo}_{\mathrm{K} \alpha}$ radiation $(\lambda=0.71073 \AA)$ and the $\omega$-scan technique. Orientation matrix and lattice parameters were obtained by least-squares refinement of the diffraction data of 34 reflections within the range of $4^{\circ}<\theta<17^{\circ}$. The index ranges of data collection were $-11 \leq \mathrm{h} \leq 11,-12 \leq \mathrm{k} \leq 12,-13 \leq 1 \leq 14$. Intensity data were collected in the $\theta$ range $2.2-25^{\circ}, 1920$ have $(\mathrm{Fo})^{2}{ }_{-} 1.5 \sigma(\mathrm{Fo})^{2}$. All the measured independent reflections were used in the analysis. The structure was solved by direct methods and refined with full-matrix least-squares technique on $F$ using the CRYSTALS ${ }^{1}$ programs. All non-hydrogen atoms were refined anisotropically. All hydrogen atoms were either set in calculated positions and isotropically refined. The values of the discrepancy indices $R 1\left(R_{\mathrm{w} 2}\right)$ for all data were 0.1043 (0.1316), whereas those listed in Table 1 correspond to the data with $I>1.5 \sigma(I)$. The final Fourier-difference map showed maximum and minimum height peaks of 0.52 and -0.53 e $\AA^{-}$

${ }^{3}$. The values of number of reflections/number of variable parameters are 254 , and those of the goodness-of-fit are 0.989. Molecular structure was drawn with the program CAMERON ${ }^{2}$ and is reported in Figure 1.

(1) Betteridge, P. W., Carruthers, J. R., Cooper, R. I., Prout, K., Watkin, D. J. (2003). J. Appl. Cryst. 36, 1487.

(2) Watkin, D. J., Prout, C. K. \& Pearce, L. J. (1996), CAMERON, Chemical Crystallography Laboratory, OXFORD, UK 
CRYSTAL DATA of $\mathbf{4 a}$ (Table2)

Formula

$\mathrm{Fw}=$

Crystal System

a $(\AA)=$

$\mathrm{b}(\AA)=$

c $(\AA)=$

$\alpha\left(^{\circ}\right)=$

$\beta\left({ }^{\circ}\right)=$

$\gamma\left({ }^{\circ}\right)=$

$\mathrm{V}\left(\AA^{3}\right)=$

$\mathrm{Z}=$

Space group

Crystal shape

Crystal colour

Linear absorption coefficient $\mu\left(\mathrm{cm}^{-1}\right)$

Density $\rho\left(\mathrm{g} \mathrm{cm}^{3}\right)$

Diffractometer

Radiation

Scan type

Scan range $\left({ }^{\circ}\right)$

$\theta$ Limits $\left({ }^{\circ}\right)$

Octants collected

$\mathrm{Nb}$ of data collected

$\mathrm{Nb}$ of unique data collected

$\mathrm{Nb}$ of unique data used for refinement

Merging R

$\mathrm{R}=\Sigma|| \mathrm{Fo}|-| \mathrm{Fc} \| / \Sigma \mid \mathrm{Fol}$

$\mathrm{RW}^{*}=\left[\Sigma \mathrm{w}(\|\mathrm{Fol}-\mid \mathrm{Fc}\|)^{2} / \Sigma \mathrm{wFo}^{2}\right]^{1 / 2}$

Absorption correction

Secondary extinction coefficient

Goodness of fit

$\mathrm{Nb}$ of variables

$\Delta \rho \min \left(\mathrm{e} / \AA^{3}\right)$

$\Delta \rho \max \left(\mathrm{e} / \AA^{3}\right)$
$\mathrm{C}_{21} \mathrm{H}_{16} \mathrm{O}_{5} \mathrm{MnS}$

435.35

triclinic

9.4006(15)

$10.4439(15)$

$11.9184(14)$

$102.274(12)$

$104.203(11)$

$112.428(14)$

985.5 (3)

2

$\mathrm{P}-1$

Parallelepiped

yellow

8.05

1.47

KAPPACCD-Enraf Nonius

$\operatorname{MoK} \alpha(\lambda=0.71073 \AA)$

$\omega / 2 \theta$

$0.8+0.345 \operatorname{tg} \theta$

2.2-25

$-11,11 ;-12,12 ;-13,14$

7835

3452

$1920(\mathrm{Fo})^{2}>1.5 \sigma(\mathrm{Fo})^{2}$

0.20

0.0540

0.0596

difabs $(\mathrm{Tmin}=0.95, \mathrm{Tmax}=1.02)$

None

0.989

254

$-0.53$

0.52

* Weighting scheme of the form $\mathrm{w}=\mathrm{w}^{\prime}\left[1-((\|\mathrm{Fo}|-| \mathrm{Fc}\|) / 6 \sigma(\mathrm{Fo}))^{2}\right]^{2}$ with $\mathrm{w}^{\prime}=1 / \Sigma_{\mathrm{r}}$ $\mathrm{A}_{\mathrm{r}} \mathrm{T}_{\mathrm{r}}(\mathrm{X})$ with coefficients $0.248,0.211$ and 0.0925 for a Chebyshev serie for which $\mathrm{X}=\mathrm{Fc} / \mathrm{Fc}(\max )$ 
Table II : Fractional atomic coordinates for $\mathrm{C}_{21} \mathrm{H}_{16} \mathrm{O}_{5} \mathrm{MnS}$

(4a)

$\begin{array}{lcccc}\text { Atom } & \mathbf{x} / \mathrm{a} & \mathrm{y} / \mathrm{b} & \mathrm{z} / \mathrm{C} & \mathrm{U} \text { (eqv) } \\ \text { Mn (1) } & 0.14436(10) & 0.00309(9) & 0.76549(8) & 0.0378 \\ \mathrm{O}(1) & 0.2494(5) & 0.3390(5) & 0.8978(4) & 0.0640 \\ O(2) & -0.0770(7) & 0.0376(7) & 0.8932(5) & 0.0906 \\ O(3) & -0.1503(6) & -0.2579(5) & 0.5796(5) & 0.0752 \\ O(4) & 0.2643(7) & -0.1542(6) & 0.9105(5) & 0.0791 \\ O(5) & 0.2077(7) & -0.1465(6) & 0.4661(4) & 0.0846 \\ C(1) & 0.3215(6) & 0.0305(5) & 0.6670(4) & 0.0345 \\ C(2) & 0.2059(6) & 0.0772(6) & 0.6222(5) & 0.0366 \\ C(3) & 0.1826(7) & 0.1824(6) & 0.6999(5) & 0.0412 \\ C(4) & 0.2839(7) & 0.2432(6) & 0.8267(5) & 0.0409 \\ C(5) & 0.3935(7) & 0.1899(6) & 0.8693(5) & 0.0396 \\ C(6) & 0.4678(6) & 0.1316(5) & 0.7836(4) & 0.0335 \\ C(7) & 0.3413(7) & -0.0825(6) & 0.5776(5) & 0.0445 \\ C(8) & 0.3696(8) & -0.1966(6) & 0.6239(5) & 0.0492 \\ C(9) & 0.5254(8) & -0.1941(8) & 0.6681(7) & 0.0671 \\ C(10) & 0.5163(16) & -0.3034(19) & 0.6933(12) & 0.1385 \\ C(11) & 0.365(2) & -0.4057(11) & 0.6778(9) & 0.1231 \\ C(12) & 0.6102(6) & 0.2512(5) & 0.7682(5) & 0.0354 \\ C(13) & 0.5822(7) & 0.3213(6) & 0.6842(5) & 0.0449 \\ C(14) & 0.7116(8) & 0.4332(7) & 0.6727(6) & 0.0558 \\ C(15) & 0.8712(9) & 0.4750(7) & 0.7454(7) & 0.0640 \\ C(16) & 0.9008(7) & 0.4059(9) & 0.8274(7) & 0.0719 \\ C(17) & 0.7703(7) & 0.2932(7) & 0.8380(5) & 0.0529 \\ C(18) & 0.3146(10) & 0.3745(9) & 1.0283(7) & 0.0854 \\ C(19) & 0.0133(8) & 0.0258(7) & 0.8463(6) & 0.0548 \\ C(20) & -0.0353(8) & -0.1573(7) & 0.6502(6) & 0.0507 \\ C(21) & 0.2145(8) & -0.0966(7) & 0.8511(5) & 0.0522 \\ \text { S(1) } & 0.2212(3) & -0.3483(2) & 0.6204(2) & 0.0989\end{array}$


Table III : Interatomic distances ( $\AA$ ) for $\mathrm{C}_{21} \mathrm{H}_{16} \mathrm{O}_{5} \mathrm{MnS}$

$\begin{array}{llll}M n(1)-C(1) & 2.230(5) & M n(1)-C(2) & 2.135(5) \\ M n(1)-C(3) & 2.120(5) & M n(1)-C(4) & 2.188(5) \\ M n(1)-C(5) & 2.211(5) & M n(1)-C(19) & 1.792(7) \\ M n(1)-C(20) & 1.808(7) & M n(1)-C(21) & 1.790(6) \\ O(1)-C(4) & 1.359(6) & O(1)-C(18) & 1.430(9) \\ O(2)-C(19) & 1.153(7) & O(3)-C(20) & 1.137(7) \\ O(4)-C(21) & 1.147(7) & O(5)-C(7) & 1.398(7) \\ C(1)-C(2) & 1.390(7) & C(1)-C(6) & 1.506(7) \\ C(1)-C(7) & 1.514(7) & C(2)-C(3) & 1.402(7) \\ C(3)-C(4) & 1.420(8) & C(4)-C(5) & 1.390(8) \\ C(5)-C(6) & 1.521(7) & C(6)-C(12) & 1.526(7) \\ C(7)-C(8) & 1.498(8) & C(8)-C(9) & 1.417(8) \\ C(8)-S(1) & 1.648(7) & C(9)-C(10) & 1.218(16) \\ C(10)-C(11) & 1.348(17) & C(11)-S(1) & 1.734(12) \\ C(12)-C(13) & 1.392(7) & C(12)-C(17) & 1.370(8) \\ C(13)-C(14) & 1.389(8) & C(14)-C(15) & 1.376(9) \\ C(15)-C(16) & 1.368(10) & C(16)-C(17) & 1.394(9)\end{array}$

Table IV : Bond angles $\left({ }^{\circ}\right)$ for $\mathrm{C}_{21} \mathrm{H}_{16} \mathrm{O}_{5} \mathrm{MnS}$

\begin{tabular}{|c|c|c|c|c|c|c|c|}
\hline$C(1)$ & $-\operatorname{Mn}(1)$ & $-C(2)$ & $37.07(18)$ & $C(1)$ & $-\operatorname{Mn}(1)$ & $-c(3)$ & $68.11(19)$ \\
\hline$C(2)$ & $-\operatorname{Mn}(1)$ & $-C(3)$ & $38.5(2)$ & $C(1)$ & $-M n(1)$ & $-C(4)$ & 78.51 (19) \\
\hline$C(2)$ & $-\operatorname{Mn}(1)$ & $-C(4)$ & $68.0(2)$ & $C(3)$ & $-M n(1)$ & $-C(4)$ & $38.5(2)$ \\
\hline$C(1)$ & $-\operatorname{Mn}(1)$ & $-C(5)$ & $64.88(18)$ & $C(2)$ & $-M n(1)$ & $-C(5)$ & $78.50(19$ \\
\hline$C(3)$ & $-\operatorname{Mn}(1)$ & $-C(5)$ & $67.9(2)$ & $C(4)$ & $-M n(1)$ & $-C(5)$ & $36.8(2)$ \\
\hline$C(1)$ & $-\operatorname{Mn}(1)$ & $-C(19)$ & $166.1(2)$ & $C(2)$ & $-\operatorname{Mn}(1)$ & $-C(19)$ & $132.6(2)$ \\
\hline$C(3)$ & $-\operatorname{Mn}(1)$ & - C (19) & $98.6(2)$ & $C(4)$ & $-\operatorname{Mn}(1)$ & - C (19) & $88.5(2)$ \\
\hline$C(5)$ & $-\operatorname{Mn}(1)$ & $-C(19)$ & $107.2(2)$ & $C(1)$ & $-\operatorname{Mn}(1)$ & $-C(20)$ & $99.9(2)$ \\
\hline$C(2)$ & $-\operatorname{Mn}(1)$ & $-C(20)$ & $89.3(2)$ & $C(3)$ & $-\operatorname{Mn}(1)$ & $-C(20)$ & $107.7(2)$ \\
\hline$C(4)$ & $-\operatorname{Mn}(1)$ & $-C(20)$ & $144.7(2)$ & $C(5)$ & $-\operatorname{Mn}(1)$ & $-C(20)$ & $164.7(2)$ \\
\hline$C(1)$ & $-\operatorname{Mn}(1)$ & $-C(21)$ & $95.1(2)$ & $C(2)$ & $-\operatorname{Mn}(1)$ & $-C(21)$ & $131.8(2)$ \\
\hline$C(3)$ & $-\operatorname{Mn}(1)$ & $-C(21)$ & $153.0(2)$ & $C(4)$ & $-M n(1)$ & $-C(21)$ & $119.7(2)$ \\
\hline$C(5)$ & $-\operatorname{Mn}(1)$ & $-C(21)$ & $86.0(2)$ & $C(19)$ & $-\operatorname{Mn}(1)$ & $-C(20)$ & $87.8(3)$ \\
\hline$C(19)$ & $-\operatorname{Mn}(1)$ & $-C(21)$ & $95.6(3)$ & $C(20)$ & $-\operatorname{Mn}(1)$ & $-C(21)$ & $95.6(3)$ \\
\hline$C(4)$ & $-O(1)$ & $-C(18)$ & $117.6(5)$ & Mn (1) & $-C(1)$ & $-C(2)$ & $67.7(3)$ \\
\hline $\mathrm{Mn}(1)$ & $-C(1)$ & $-C(6)$ & $92.5(3)$ & $C(2)$ & $-C(1)$ & $-C(6)$ & $119.0(4)$ \\
\hline $\operatorname{Mn}(1)$ & $-C(1)$ & $-C(7)$ & $130.9(4)$ & $C(2)$ & $-C(1)$ & $-C(7)$ & $118.9(4)$ \\
\hline$C(6)$ & $-C(1)$ & $-C(7)$ & $117.2(4)$ & Mn (1) & $-C(2)$ & $-C(1)$ & $75.2(3)$ \\
\hline $\mathrm{Mn}(1)$ & $-C(2)$ & $-C(3)$ & $70.2(3)$ & $C(1)$ & $-C(2)$ & $-C(3)$ & $121.6(5)$ \\
\hline $\mathrm{Mn}(1)$ & $-c(3)$ & $-C(2)$ & $71.3(3)$ & Mn (1) & $-c(3)$ & $-C(4)$ & $73.4(3)$ \\
\hline$C(2)$ & $-C(3)$ & $-C(4)$ & $117.9(5)$ & Mn (1) & $-C(4)$ & $-O(1)$ & $124.6(4)$ \\
\hline $\mathrm{Mn}(1)$ & $-C(4)$ & $-c(3)$ & $68.2(3)$ & $O(1)$ & $-C(4)$ & $-c(3)$ & $114.8(5)$ \\
\hline $\mathrm{Mn}(1)$ & $-C(4)$ & $-C(5)$ & $72.5(3)$ & $O(1)$ & $-C(4)$ & $-C(5)$ & $125.8(5)$ \\
\hline$C(3)$ & $-C(4)$ & $-C(5)$ & $119.0(5)$ & Mn (1) & $-C(5)$ & $-C(4)$ & $70.7(3)$ \\
\hline $\mathrm{Mn}(1)$ & $-C(5)$ & $-C(6)$ & $92.9(3)$ & $C(4)$ & $-C(5)$ & $-C(6)$ & $119.6(4)$ \\
\hline$C(1)$ & $-C(6)$ & $-C(5)$ & $103.8(4)$ & $C(1)$ & $-C(6)$ & $-C(12)$ & $115.5(4)$ \\
\hline$C(5)$ & $-C(6)$ & $-C(12)$ & $114.3(4)$ & $O(5)$ & $-C(7)$ & $-C(1)$ & $109.3(4)$ \\
\hline$O(5)$ & $-C(7)$ & $-C(8)$ & $111.8(5)$ & $C(1)$ & $-C(7)$ & $-C(8)$ & $115.8(4)$ \\
\hline$C(7)$ & $-C(8)$ & $-C(9)$ & $124.7(6)$ & $C(7)$ & $-C(8)$ & $-s(1)$ & $124.2(4)$ \\
\hline$C(9)$ & $-C(8)$ & $-s(1)$ & $110.9(5)$ & $C(8)$ & $-C(9)$ & $-C(10)$ & $112.9(9)$ \\
\hline$C(9)$ & $-C(10)$ & $-C(11)$ & $117.4(12)$ & $C(10)$ & $-C(11)$ & $-S(1)$ & $108.7(8)$ \\
\hline$C(6)$ & $-C(12)$ & $-C(13)$ & $121.5(5)$ & $C(6)$ & $-C(12)$ & - C (17) & $120.5(5)$ \\
\hline$C(13)$ & $-C(12)$ & $-C(17)$ & $118.1(5)$ & $C(12)$ & $-C(13)$ & $-C(14)$ & $121.5(5)$ \\
\hline$C(13)$ & $-C(14)$ & $-C(15)$ & $119.2(6)$ & $C(14)$ & $-C(15)$ & $-C(16)$ & $119.9(6)$ \\
\hline$C(15)$ & - C (16) & $-C(17)$ & $120.5(6)$ & $C(12)$ & - C (17) & $-C(16)$ & $120.7(6)$ \\
\hline Mn (1) & $-C(19)$ & $-O(2)$ & $176.9(6)$ & Mn (1) & $-C(20)$ & $-o(3)$ & $178.4(5)$ \\
\hline $\mathrm{Mn}(1)$ & $-C(21)$ & $-O(4)$ & $176.7(6)$ & $C(8)$ & $-S(1)$ & $-C(11)$ & $90.1(5)$ \\
\hline
\end{tabular}


Table S1 : Anisotropic thermal parameters for $\mathrm{C}_{21} \mathrm{H}_{16} \mathrm{O}_{5} \mathrm{MnS}$

\begin{tabular}{|c|c|c|c|c|c|c|}
\hline Atom & $u(11)$ & $u(22)$ & $u(33)$ & $u(23)$ & $u(13)$ & $u(12)$ \\
\hline $\operatorname{Mn}(1)$ & $0.0370(5)$ & $0.0395(5)$ & $0.0368(5)$ & $0.0142(4)$ & $0.0130(4)$ & $0.0170(4)$ \\
\hline$O(1)$ & $0.070(3)$ & $0.052(3)$ & $0.065(3)$ & $-0.001(2)$ & $0.023(2)$ & $0.035(2)$ \\
\hline$O(3)$ & $0.058(3)$ & $0.054(3)$ & $0.073(3)$ & $0.015(3)$ & $0.007(3)$ & $0.000(3)$ \\
\hline$O(4)$ & $0.099(4)$ & $0.085(4)$ & $0.074(3)$ & $0.053(3)$ & $0.029(3)$ & $0.050(3)$ \\
\hline$O(5)$ & $0.120(4)$ & $0.090(4)$ & $0.038(2)$ & $-0.002(2)$ & $-0.003(3)$ & $0.074(3)$ \\
\hline$C(2)$ & 0.039 (3) & $0.040(3)$ & $0.031(3)$ & $0.017(2)$ & $0.008(2)$ & 0.019 (3) \\
\hline$C(3)$ & $0.043(3)$ & $0.042(3)$ & $0.048(3)$ & $0.022(3)$ & $0.016(3)$ & $0.025(3)$ \\
\hline$C(4)$ & $0.046(3)$ & $0.032(3)$ & $0.040(3)$ & $0.008(3)$ & $0.012(3)$ & $0.018(3)$ \\
\hline$C(5)$ & $0.041(3)$ & $0.038(3)$ & $0.030(3)$ & $0.007(2)$ & $0.007(2)$ & 0.015 (3) \\
\hline$C(6)$ & $0.036(3)$ & $0.032(3)$ & $0.030(3)$ & $0.010(2)$ & $0.007(2)$ & $0.017(2)$ \\
\hline$C(7)$ & $0.049(3)$ & $0.040(3)$ & $0.038(3)$ & $0.005(3)$ & $0.015(3)$ & $0.019(3)$ \\
\hline$C(8)$ & $0.066(4)$ & $0.047(3)$ & $0.043(3)$ & $0.012(3)$ & $0.020(3)$ & $0.036(3)$ \\
\hline$C(10)$ & $0.121(10)$ & $0.160(13)$ & $0.118(9)$ & $0.032(9)$ & $0.017(8)$ & $0.072(10)$ \\
\hline$C(11)$ & $0.257(15)$ & $0.081(7)$ & $0.104(7)$ & $0.057(6)$ & $0.078(9)$ & $0.126(9)$ \\
\hline$C(12)$ & $0.037(3)$ & $0.031(3)$ & $0.032(3)$ & $0.001(2)$ & $0.010(2)$ & $0.015(2)$ \\
\hline$C(13)$ & $0.051(4)$ & $0.038(3)$ & $0.043(3)$ & $0.012(3)$ & $0.016(3)$ & 0.019 (3) \\
\hline$C(14)$ & $0.060(4)$ & $0.040(4)$ & $0.061(4)$ & $0.016(3)$ & $0.023(4)$ & $0.018(3)$ \\
\hline$C(15)$ & $0.065(5)$ & $0.049(4)$ & $0.066(4)$ & $0.010(4)$ & $0.036(4)$ & $0.012(4)$ \\
\hline$C(16)$ & $0.029(4)$ & $0.086(5)$ & $0.068(5)$ & $0.003(4)$ & $0.011(3)$ & $0.010(4)$ \\
\hline$C(17)$ & $0.038(4)$ & $0.067(4)$ & $0.049(4)$ & $0.016(3)$ & $0.010(3)$ & $0.025(3)$ \\
\hline$C(18)$ & $0.092(6)$ & $0.084(6)$ & $0.061(5)$ & $-0.012(4)$ & $0.030(4)$ & $0.039(5)$ \\
\hline$C(19)$ & $0.054(4)$ & $0.058(4)$ & $0.052(4)$ & $0.022(3)$ & $0.020(3)$ & $0.023(3)$ \\
\hline$C(20)$ & $0.044(4)$ & $0.052(4)$ & $0.058(4)$ & $0.025(4)$ & $0.023(3)$ & $0.018(3)$ \\
\hline$C(21)$ & $0.058(4)$ & $0.054(4)$ & $0.046(4)$ & $0.025(3)$ & $0.022(3)$ & $0.022(3)$ \\
\hline$S(1)$ & 0.1155 (19) & 0.0591 (13) & $0.123(2)$ & $0.0310(13)$ & $0.0671(16)$ & $0.0268(13)$ \\
\hline
\end{tabular}

$\mathrm{C}_{21} \mathrm{H}_{16} \mathrm{O}_{5} \mathrm{MnS}$

Table S2 : Hydrogen atoms fractional atomic coordinates for

\begin{tabular}{|c|c|c|c|c|}
\hline Atom & $x / a$ & $y / b$ & $z / c$ & U (iso) \\
\hline $\mathrm{H}(21)$ & 0.1366 & 0.0338 & 0.5317 & $0.122(8)$ \\
\hline H (31) & 0.0973 & 0.2143 & 0.6675 & $0.122(8)$ \\
\hline H (51) & 0.4231 & 0.1900 & 0.9562 & $0.122(8)$ \\
\hline H (61) & 0.5263 & 0.0772 & 0.8171 & $0.122(8)$ \\
\hline $\mathrm{H}(71)$ & 0.4483 & -0.0260 & 0.5667 & $0.122(8)$ \\
\hline Н $(91)$ & 0.6324 & -0.1087 & 0.6773 & $0.122(8)$ \\
\hline $\mathrm{H}(101)$ & 0.6223 & -0.3170 & 0.7208 & $0.122(8)$ \\
\hline $\mathrm{H}(111)$ & 0.3443 & -0.4982 & 0.6969 & $0.122(8)$ \\
\hline $\mathrm{H}(131)$ & 0.4644 & 0.2899 & 0.6309 & $0.122(8)$ \\
\hline H (141) & 0.6891 & 0.4839 & 0.6117 & $0.122(8)$ \\
\hline H (151) & 0.9680 & 0.5567 & 0.7394 & $0.122(8)$ \\
\hline $\mathrm{H}(161)$ & 1.0184 & 0.4362 & 0.8818 & $0.122(8)$ \\
\hline Н (171) & 0.7935 & 0.2409 & 0.8975 & $0.122(8)$ \\
\hline $\mathrm{H}(181)$ & 0.2825 & 0.4443 & 1.0729 & $0.122(8)$ \\
\hline H (182) & 0.2721 & 0.2806 & 1.0503 & $0.122(8)$ \\
\hline H (183) & 0.4392 & 0.4183 & 1.0598 & $0.122(8)$ \\
\hline
\end{tabular}

